\title{
Prevalence of abnormal cardiovascular magnetic resonance findings in recovered patients from COVID-19: a systematic review and meta-analysis
}

\author{
Jin Young $\operatorname{Kim}^{1}$ (1), Kyunghwa Han² ${ }^{2}$ and Young Joo Suh ${ }^{2 *}$
}

\begin{abstract}
Background: The prevalence of abnormal cardiovascular magnetic resonance (CMR) findings in recovered coronavirus disease 2019 (COVID-19) patients is unclear. This study aimed to investigate the prevalence of abnormal CMR findings in recovered COVID-19 patients.

Methods: A systematic literature search was performed to identify studies that report the prevalence of abnormal CMR findings in recovered COVID-19 patients. The number of patients with abnormal CMR findings and diagnosis of myocarditis on CMR (based on the Lake Louise criteria) and each abnormal CMR parameter were extracted. Subgroup analyses were performed according to patient characteristics (athletes vs. non-athletes and normal vs. undetermined cardiac enzyme levels). The pooled prevalence and 95\% confidence interval (Cl) of each CMR finding were calculated. Study heterogeneity was assessed, and meta-regression analysis was performed to investigate factors associated with heterogeneity.
\end{abstract}

Results: In total, 890 patients from 16 studies were included in the analysis. The pooled prevalence of one or more abnormal CMR findings in recovered COVID-19 patients was 46.4\% (95\% Cl 43.2\%-49.7\%). The pooled prevalence of myocarditis and late gadolinium enhancement (LGE) was 14.0\% (95\% Cl 11.6\%-16.8\%) and 20.5\% (95\% Cl 17.7\%$23.6 \%)$, respectively. Further, heterogeneity was observed $\left(1^{2}>50 \%, p<0.1\right)$. In the subgroup analysis, the pooled prevalence of abnormal CMR findings and myocarditis was higher in non-athletes than in athletes (62.5\% vs. 17.1\% and $23.9 \%$ vs. $2.5 \%$, respectively). Similarly, the pooled prevalence of abnormal CMR findings and LGE was higher in the undetermined than in the normal cardiac enzyme level subgroup $(59.4 \%$ vs. $35.9 \%$ and $45.5 \%$ vs. $8.3 \%$, respectively). Being an athlete was a significant independent factor related to heterogeneity in multivariate meta-regression analysis $(p<0.05)$.

Conclusions: Nearly half of recovered COVID-19 patients exhibited one or more abnormal CMR findings. Athletes and patients with normal cardiac enzyme levels showed a lower prevalence of abnormal CMR findings than nonathletes and patients with undetermined cardiac enzyme levels.

\footnotetext{
*Correspondence: rongzusuh@gmail.com

2 Department of Radiology, Research Institute of Radiological Science,

Severance Hospital, Yonsei University College of Medicine, 50-1 Yonsei-ro, Seodaemun-gu, Seoul 03722, Korea

Full list of author information is available at the end of the article
}

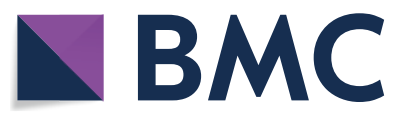

Auth 2021. Open Access This article is licensed under a Creative Commons Attribution 4.0 International License, which permits use, sharing, adaptation, distribution and reproduction in any medium or format, as long as you give appropriate credit to the original author(s) and the source, provide a link to the Creative Commons licence, and indicate if changes were made. The images or other third party material in this article are included in the article's Creative Commons licence, unless indicated otherwise in a credit line to the material. If material is not included in the article's Creative Commons licence and your intended use is not permitted by statutory regulation or exceeds the permitted use, you will need to obtain permission directly from the copyright holder. To view a copy of this licence, visit http://creativecommons.org/licenses/by/4.0/. The Creative Commons Public Domain Dedication waiver (http://creativeco mmons.org/publicdomain/zero/1.0/) applies to the data made available in this article, unless otherwise stated in a credit line to the data. 
Trial registration The study protocol was registered in the PROSPERO database (registration number: CRD42020225234).

Keywords: Cardiac magnetic resonance imaging, Magnetic resonance imaging, Coronavirus disease 2019

\section{Background}

The spread of coronavirus disease 2019 (COVID-19) was rapid, and COVID-19 was quickly designated as a pandemic since the first identified case in December 2019 in Wuhan, China [1]. As of July 7, 2021, more than 184 million people have been diagnosed with COVID-19 and nearly 4 million have died of the infection [2]. Although COVID-19 is primarily a respiratory disease, cardiovascular complications have been reported [3, 4] and are associated with higher mortality and risk of severe COVID-19 [5, 6]. Cardiac involvement in COVID-19 can manifest as myocarditis, heart failure, acute coronary syndrome, or arrhythmias [4, 7]. Among these, myocarditis has clinical significance because myocardial inflammation can result in permanent myocardial damage and contribute to the development of arrhythmia or chronic heart failure $[7,8]$.

Cardiovascular magnetic resonance (CMR) is used to diagnose cardiovascular complications of COVID-19, such as acute myocarditis, using the recently updated Lake Louise criteria [9]. Individual reports and one systematic review of CMR findings in COVID-19 patients have been published to date; however, most focused on patients in the active disease stage [10]. Notably, recent data indicated that the prevalence of abnormal CMR findings, such as myocardial edema and late gadolinium enhancement (LGE), in recovered COVID-19 patients is substantial [11-22]; however, their prevalence is highly variable. Although the clinical significance of abnormal CMR findings in recovered COVID-19 patients is not yet fully understood, determining the prevalence of such findings in certain subgroups of patients would benefit clinical decision-making. For example, the presence of myocardial scars after myocarditis can lead to sudden cardiac death, especially in athletes. Consequently, the prevalence of abnormal CMR findings in athletes who have recovered from COVID-19 affects their return to play [23-25].

Therefore, the purpose of this study was to investigate the prevalence of abnormal CMR findings in recovered COVID-19 patients through meta-analysis.

\section{Methods}

Our methods followed the recommendations of the preferred reporting items for systematic reviews and metaanalyses statement [26], and the study protocol was registered in the PROSPERO database (registration number: CRD42020225234).

\section{Literature search}

Two cardiothoracic radiologists with 5 and 8 years of experience, in performing meta-analyses designed the search strategy in consensus. Each individual independently performed systematic searches of PubMed, EMBASE, the Cochrane library, SSRN, and MedRxiv/ BioRxiv on March 3, 2021, to identify studies published since 2020. The search terms are listed in Additional file 1: Appendix S1.

\section{Study selection}

Two investigators independently reviewed the retrieved articles. A flowchart summarizing the literature search process is shown in Fig. 1. To determine the study eligibility, the full text of articles was evaluated for inclusion using the following criteria: (1) type of study, i.e., randomized controlled studies, prospective or retrospective cohort studies, and case-control studies with more than 10 patients; (2) study population, i.e., patients who recovered from COVID-19 and underwent CMR after recovery; and (3) primary outcome, i.e., the prevalence of abnormal CMR findings. Abnormal CMR findings included the presence of ventricular systolic dysfunction on cine imaging, the presence of myocardial or pericardial late gadolinium enhancement (LGE), abnormal signal intensity on $\mathrm{T} 2$-weighted (T2w) imaging, elevated native $\mathrm{T} 1$ or $\mathrm{T} 2$ values on the mapping sequence, a diagnosis of myocarditis based on the updated Lake Louise criteria, and the presence of pericardial effusion [9].

In contrast, a study was excluded if the study population was restricted to COVID-19 patients with multisystem inflammatory syndrome or reported CMR findings during the acute stage of COVID-19.

\section{Data extraction}

Two investigators independently extracted data with disagreements resolved by consensus. The extracted parameters included the following: (a) article information and patient characteristics; (b) CMR protocol, i.e., CMR scanner type (1.5 or $3 \mathrm{~T}$ ) and obtained CMR sequences including cine, parametric mapping (T1 and T2), LGE, and T2w; and (c) CMR findings, i.e., the number of patients with normal and abnormal CMR findings, abnormal cine findings (ventricular systolic dysfunction), 


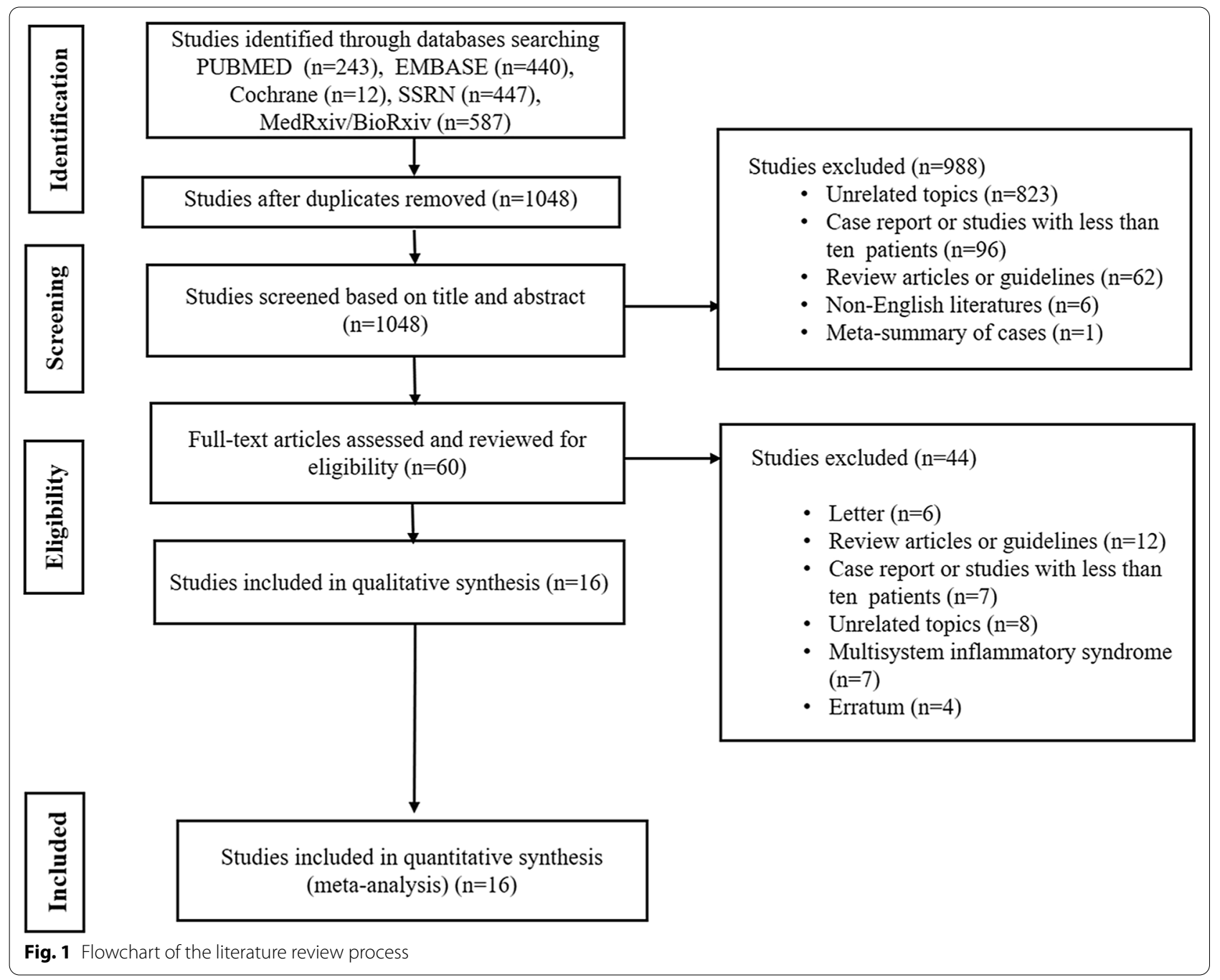

elevated parametric mapping (native $\mathrm{T} 1$ and $\mathrm{T} 2$ ) and extracellular volume (ECV) values, presence of LGE (myocardial or pericardial), myocardial segments with abnormal T2 or LGE areas, myocardial LGE patterns (non-ischemic, ischemic, or dual) that fulfilled the diagnostic criteria for myocarditis on CMR based on the Lake Louise criteria [9], and presence of pericardial effusion. LGE at the right ventricular (RV) insertion points in the interventricular septum was not considered to indicate LGE presence because it is a common non-specific finding in athletes [27].

\section{Subgroup analysis}

Subgroups were stratified according to (a) whether a patient group was limited to athletes and (b) levels of cardiac enzymes (troponin I or high-sensitivity troponin $\mathrm{T}$ ) when CMR was performed. Studies wherein the cardiac enzyme data were not extractable were assigned to the "undetermined cardiac enzyme level" subgroup. An analysis of an "elevated cardiac enzyme level" subgroup could not be performed, because there were only seven patients in three studies who had elevated cardiac enzyme levels and extractable CMR findings [11, 28, 29].

\section{Quality assessment}

Two investigators independently performed quality assessments of the selected studies using the NewcastleOttawa Quality Scale [30]: for each question within the Selection and Exposure/Outcome categories, the maximum score is 1 , and for the Comparability category, the top score is 2. A study with a total score of 6 or higher was considered of "high quality."

\section{Statistical analysis}

The pooled prevalence and 95\% confidence interval (CI) of each CMR finding were estimated using a generalized 
linear mixed model. The heterogeneity between studies was assessed using chi-square-based $Q$ statistics and $\mathrm{I}^{2}$ statistics [31, 32], and significant heterogeneity was defined as a P-value of $<0.1$ or an $\mathrm{I}^{2}$ value of $>50 \%$. Subgroup analysis of the prevalence of CMR findings was performed for the "athlete" versus (vs.) "non-athlete" subgroups and the "normal cardiac enzyme level" vs. "undetermined cardiac enzyme level" subgroups. Meta-regression analysis was performed for major CMR parameters to investigate their contribution to a study's heterogeneity, using the covariates "athlete" and "undetermined cardiac enzyme level." Variables with P-values of $<0.2$ in the univariable meta-regression analysis were included in the multivariable analysis. A P-value of $<0.05$ was considered to indicate a statistically significant difference in the multivariable analysis. Publication biases were drawn as funnel plots and evaluated using the Egger test [33]. The analysis was performed using $\mathrm{R}$ (version 4.0.3; R Foundation for Statistical Computing, Vienna, Austria) with the "metafor" and "meta" packages [34, 35].

\section{Results}

\section{Study characteristics}

Following the literature search, 890 patients from 16 studies were included in this meta-analysis [11-14, 1622, 28, 29, 36, 37]. Tables 1 and 2 summarize the study characteristics and CMR protocols of the included studies, respectively. A greater percentage of the included studies were conducted retrospectively $(62.5 \%)$ at a single institution (93.8\%). Most studies (81.3\%) obtained cine, parametric mapping (native T1 and T2), and LGE sequences [11-14, 16-19, 21, 22, 28, 36, 37]. Similarly, nine studies obtained T2w sequences $[11,12,16,17,20$, $21,28,29,36]$, and one study obtained a non-contrastenhanced CMR without an LGE sequence [17].

Six of the 16 included studies enrolled only athletes as participants $[16,19,21,28,36,37]$, whereas there was no restriction on the occupation of study participants in the other 10 studies [11-18, 20, 29]. Eight studies had populations with normal cardiac enzyme levels $[11,12,15$, $16,19,28,29,37]$. Seven other studies had patients with undetermined cardiac enzyme levels $[13,14,17,18,20-$ $22]$, and one study reported data for normal and undetermined cardiac enzyme level subgroups [36].

\section{Pooled prevalence of abnormal CMR findings}

The pooled prevalence values of abnormal CMR findings are summarized in Table 3 and Fig. 2. The overall prevalence of any abnormal CMR finding in recovered COVID-19 patients was $46.4 \%$ (95\% CI 43.2\%-49.7\%) in 16 studies [11-22, 28, 29, 36, 37]. The pooled prevalence of a CMR diagnosis of myocarditis was $14.0 \%$ (95\% CI $11.6 \%-16.8 \%)$ in 12 studies [11-14, 16, 19, 21,
$22,28,29,36,37]$. The pooled prevalence of pericardial and myocardial LGE was 5.0\% (95\% CI 3.8\%-6.7\%) in 14 studies [11-16, 18-21, 28, 29, 36, 37] and 20.7\% (95\% CI $18.1 \%-23.5 \%$ ) in 15 studies [11-16, 18-22, 28, 29, 36, 37], respectively. The pooled prevalence of total (pericardial or myocardial) LGE was $20.5 \%$ (95\% CI $17.7 \%-23.6 \%$ ) in 13 studies [11-16, 19, 20, 22, 28, 29, 36, 37].

The pooled prevalence of an elevated native T1 was $26.3 \%$ (95\% CI $23.1 \%-29.8 \%$ ) in 10 studies [11, 14, 16-19, $21,22,28,36]$ and that of a T2 abnormality (increased T2 value on the T2 map or abnormal SI on T2 weighted (T2w) imaging was $16.9 \%(95 \%$ CI $14.3 \%-19.8 \%)$ in 12 studies $[11-14,16-19,21,22,28,36]$. The pooled prevalence of a T2 abnormality without LGE was $4.0 \%$ (95\% CI $2.3 \%-6.7 \%$ ) in eight studies [12, 13, 16, 19, 21, 22, 28, 36], and that of LGE without a T2 abnormality was 4.0\% (95\% CI 2.3\%-7.0\%) in seven studies [12, 16, 19, 21, 22, 28, 29]. The pooled prevalence of pericardial effusion was $15.7 \%$ (95\% CI 13.2\%-18.5\%) in 11 studies [11-14, 16, 18, 19, $21,22,28,36]$, and that of ventricular systolic dysfunction on cine CMR was $4.7 \%$ (95\% CI 3.3\%-6.6\%) in 10 studies $[11,13,14,16,19,21,28,29,36,37]$. Significant heterogeneities among the included studies were observed for all parameters of abnormal findings $\left(\mathrm{I}^{2}>50 \%\right)$.

\section{Prevalence of abnormal CMR findings relative to patient characteristics}

The pooled prevalence values of abnormal CMR findings within subgroups are summarized in Table 3.

\section{Non-athletes vs. athletes}

Of the 890 patients in 16 studies, 316 (35.5\%) subjects were athletes $[16,19,21,28,36,37]$. The pooled prevalence of abnormal CMR findings and a CMR diagnosis of myocarditis was higher in non-athletes than in athletes $(62.5 \%$ vs. $17.1 \%$ and $23.9 \%$ vs. $2.5 \%$, respectively). Similarly, compared with athletes, non-athletes had a higher pooled prevalence of other CMR abnormalities, including myocardial LGE ( $28.8 \%$ vs. $6.7 \%)$, an elevated native T1 (39.8\% vs. $4.4 \%)$, a T2 abnormality (22.9\% vs. $4.4 \%)$, a T2 abnormality without LGE (12.9\% vs. $1.6 \%)$, pericardial effusion ( $17.3 \%$ vs. $12.8 \%)$, and ventricular systolic dysfunction ( $7.4 \%$ vs. $1.3 \%)$. In contrast, the pooled prevalence values were slightly higher in athletes than in non-athletes for pericardial LGE (6.7\% vs. $4.1 \%)$ and were similar in both groups for myocardial LGE without T2 abnormality (4.1\% vs. $3.8 \%)$. After subgroup analysis, the heterogeneity of studies became insignificant for abnormal CMR and ventricular dysfunction in both subgroups and the presence of myocardial LGE without T2 abnormality in the non-athlete subgroup (all, $\mathrm{p}>0.1, \mathrm{I}^{2}<50 \%$ ). 


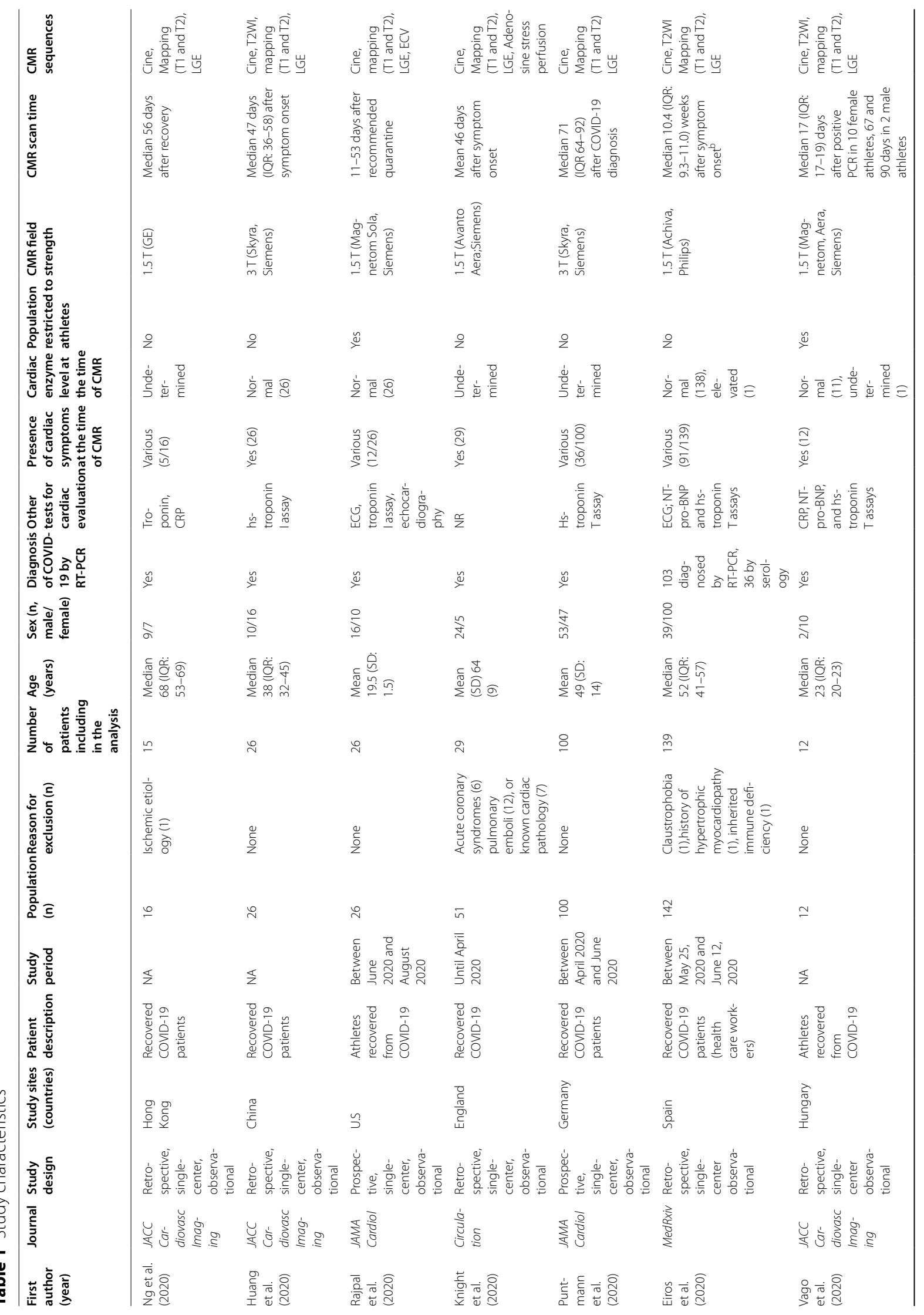




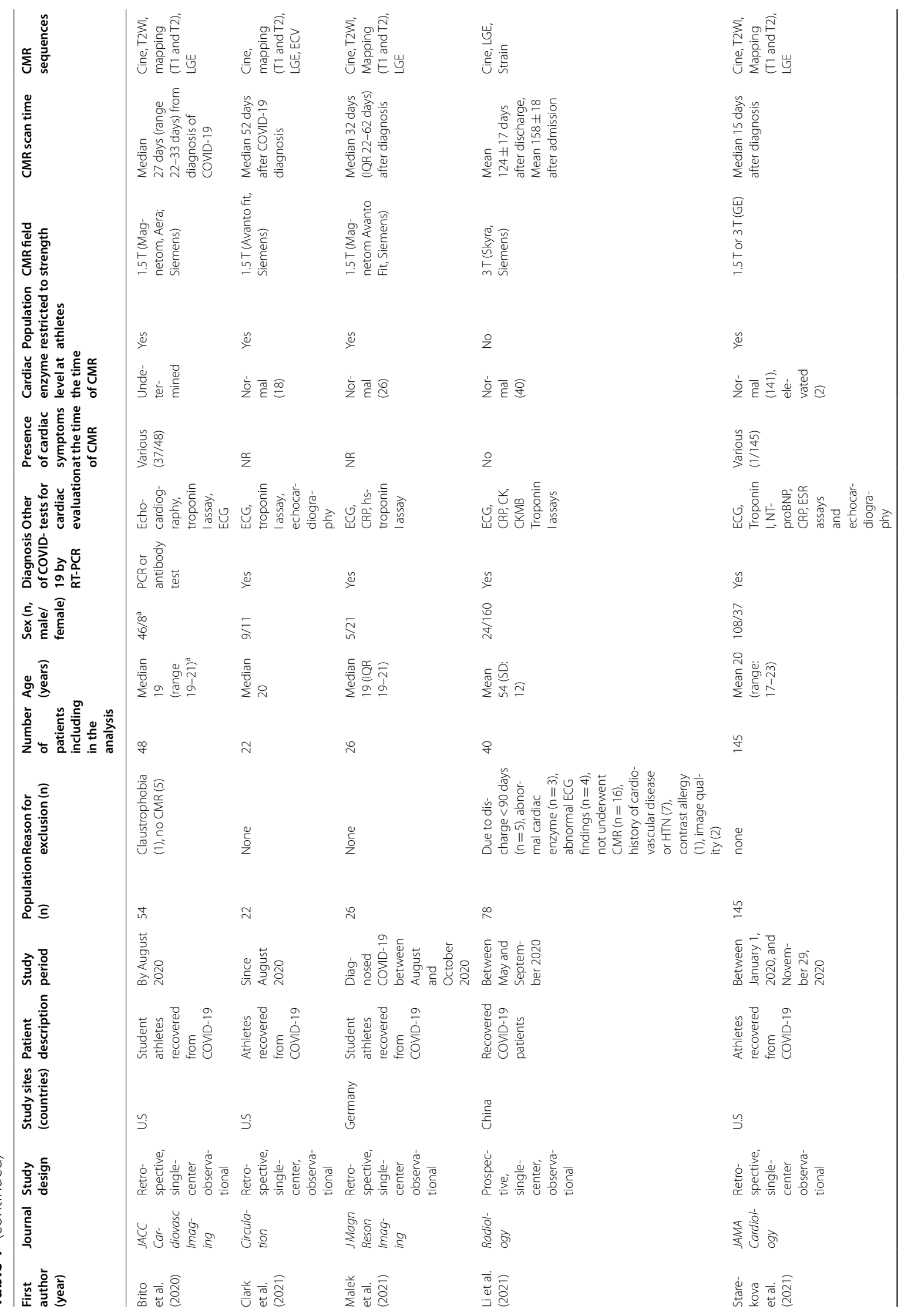




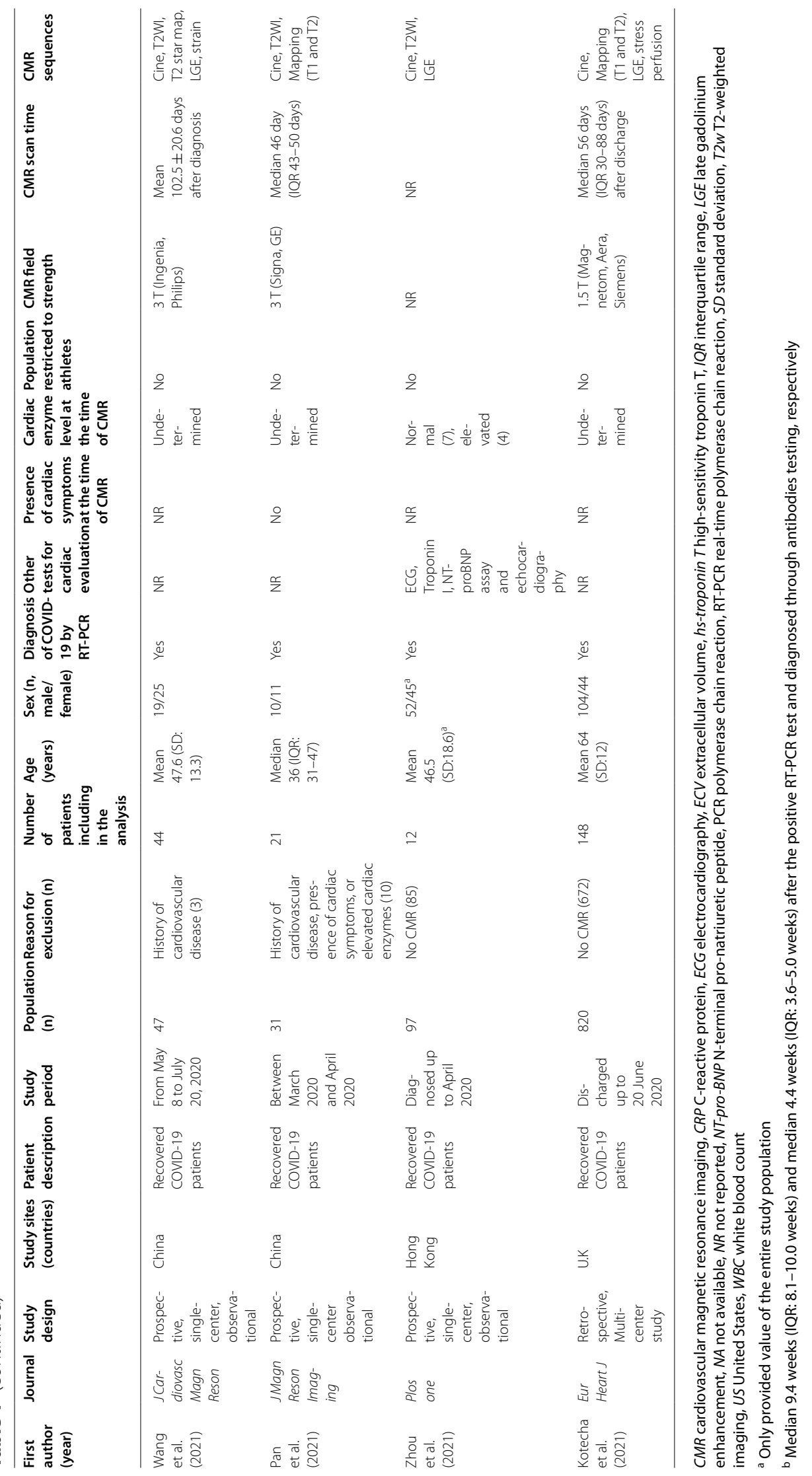




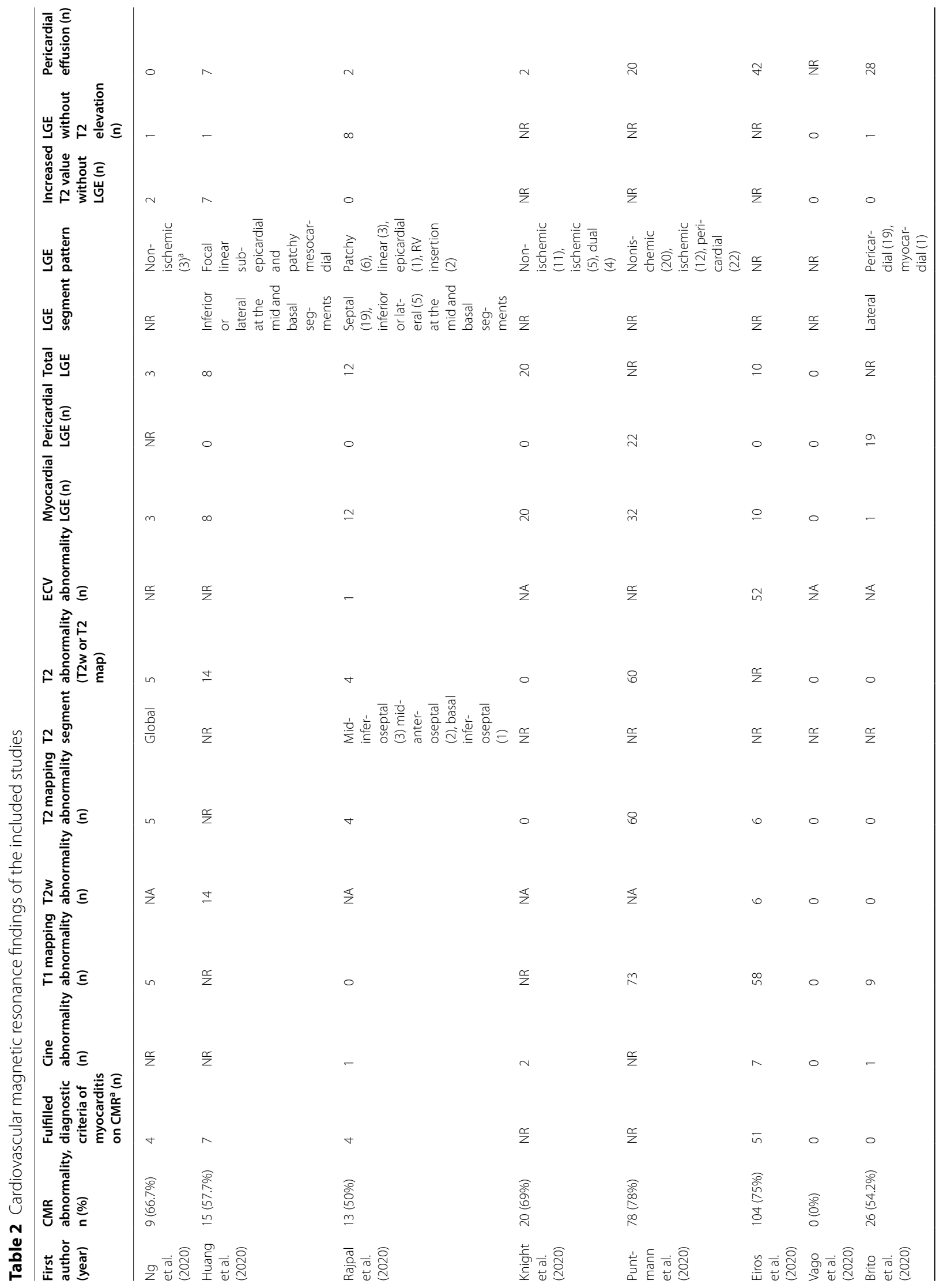




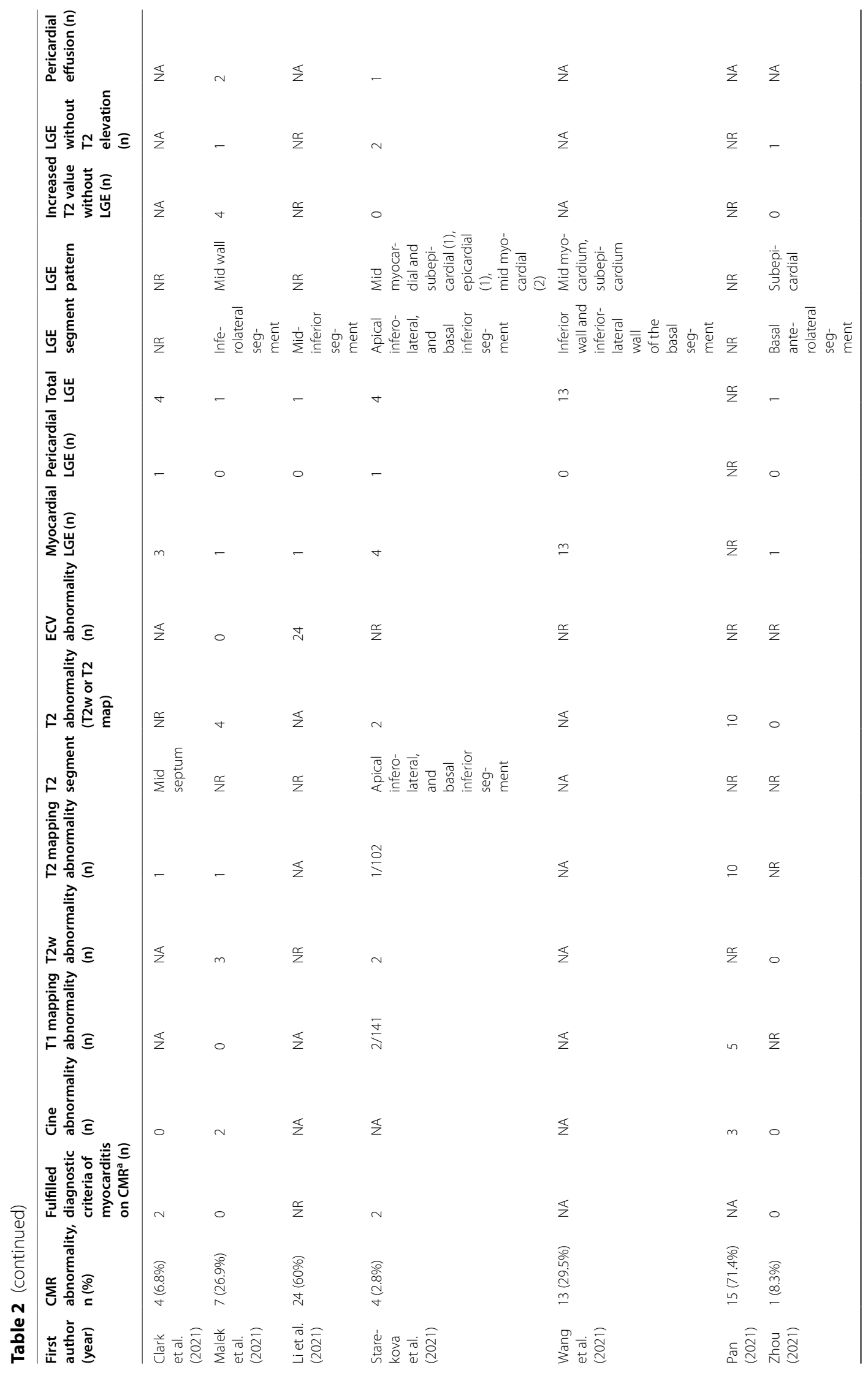




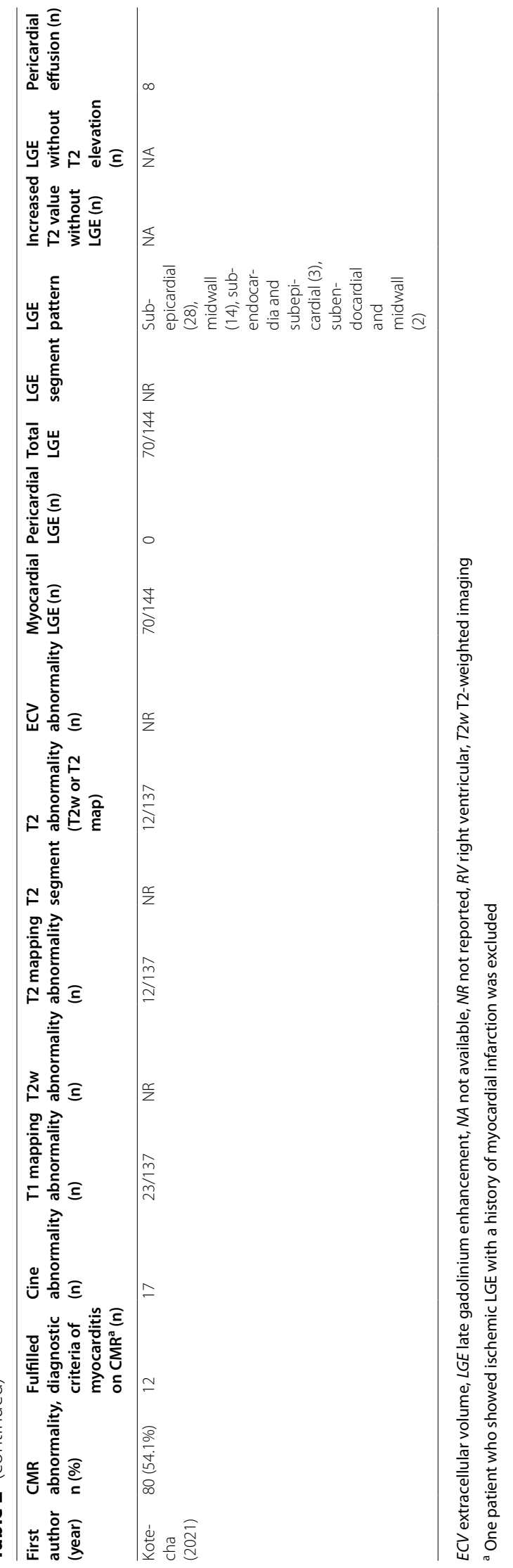




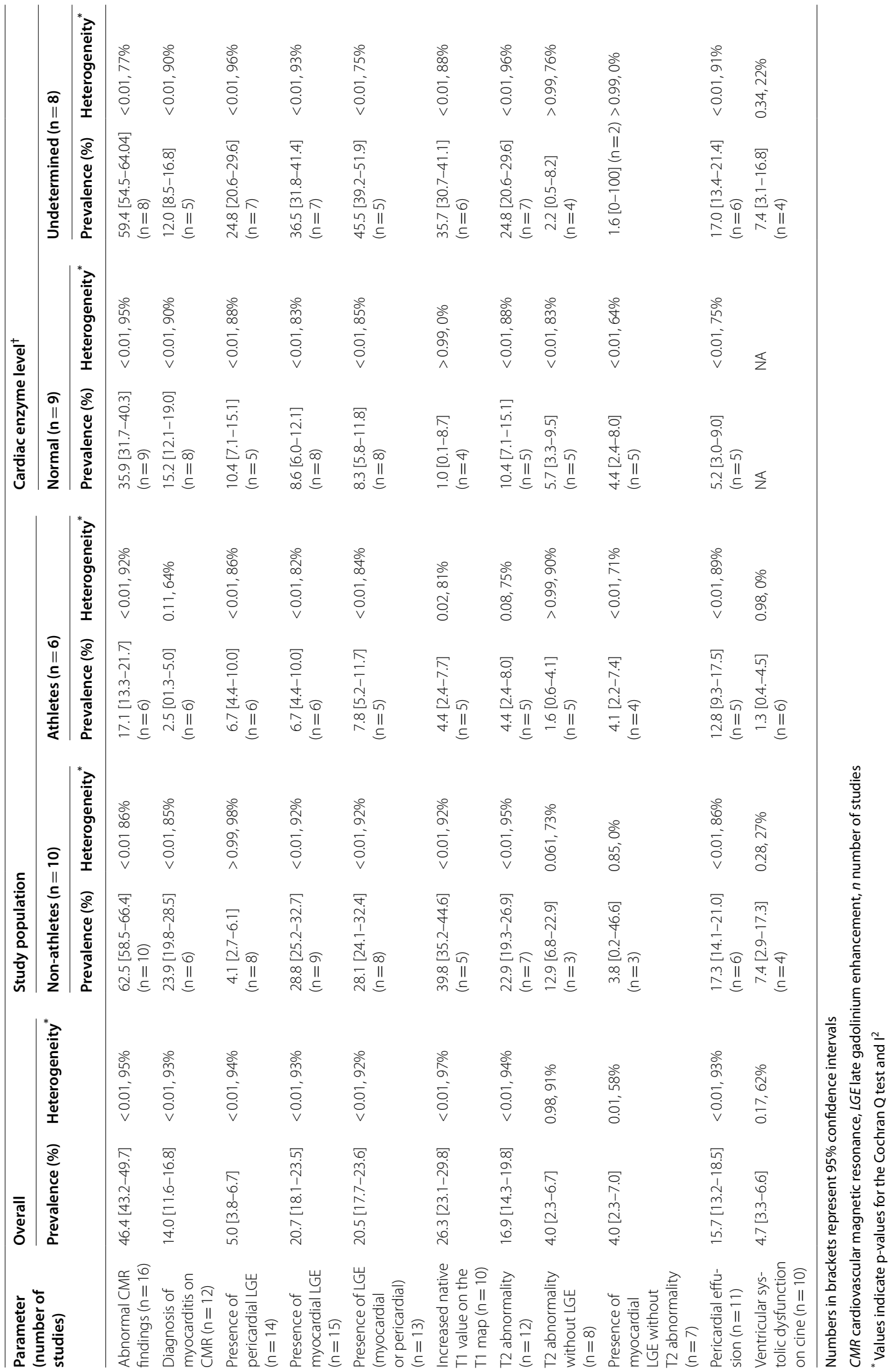




\section{Normal cardiac enzyme level vs. undetermined cardiac enzyme level}

Among the 890 patients in 16 studies, 474 (53.3\%) from nine studies [11, 12, 15, 16, 19, 28, 29, 36, 37] had normal enzyme levels (e.g. troponin) and 406 (45.6\%) from eight studies had undetermined cardiac enzyme levels [13, 14, 17, 18, 20-22, 36]. The undetermined cardiac enzyme level subgroup exhibited a higher pooled prevalence than the normal cardiac enzyme level subgroup for abnormal CMR findings (59.4\% vs. $35.9 \%$ ), the presence of pericardial (24.8\% vs. $10.4 \%)$ or myocardial LGE (36.5\% vs. $8.6 \%)$, an elevated native $\mathrm{T} 1$ value $(35.7 \%$ vs. $1 \%)$, T2 abnormality (24.8\% vs. $10.4 \%)$, and pericardial effusion ( $17 \%$ vs. $5.2 \%)$. In contrast, the pooled prevalence values were higher in the normal cardiac enzyme level subgroup than in the undetermined cardiac enzyme level subgroup for a diagnosis of myocarditis on CMR (15.2\% vs. 12.0\%) and the presence of myocardial LGE without T2 abnormality (4.4\% vs. $1.6 \%)$. After subgroup analysis, the heterogeneity between studies became insignificant for ventricular dysfunction in the undetermined enzyme level subgroup $\left(\mathrm{p}=0.34, \mathrm{I}^{2}=22 \%\right)$.

Meta-regression analysis results are summarized in Table 4. In the univariable meta-regression analyses, the athlete subgroup was significantly associated with heterogeneity for abnormal CMR findings, myocarditis diagnosis on CMR, myocardial LGE, and a T2 abnormality (all, $\mathrm{p}<0.2$ ). In contrast, undetermined cardiac enzyme level was significantly associated with heterogeneity for abnormal CMR findings and the presence of myocardial LGE (all, $\mathrm{p}<0.2$ ). In the multivariable meta-regression analyses, being an athlete was a significant independent factor associated with heterogeneity for abnormal CMR findings $(\mathrm{p}<0.05)$. However, undetermined cardiac enzyme levels were not significantly associated with heterogeneity in multivariable meta-regression analyses.

\section{Quality of the studies}

The quality assessments of the included studies are summarized in Additional file 1: Table S1. Most studies were classified as "high quality" (87.5\% of the studies received scores of 6 or 7 , and $12.5 \%$ received a score of 5).

\section{Systematic review of the ECV, patterns of LGE, and cine findings ECV findings}

Six studies reported that ECV was significantly higher in recovered COVID-19 patients than in healthy controls $[11,12,15,16,19,37]$. Huang et al. showed that ECV was significantly higher in recovered COVID-19 patients who showed abnormal CMR findings than in controls (median ECV: $28.2 \%$ vs. 23.7\%, p =0.001) [12]. Eiros et al. reported that the prevalence of elevated ECV was $37.4 \%$ $(52 / 139)$ in recovered COVID-19 patients [11]. Li et al. reported that ECV was significantly elevated in patients recovered from moderate (median ECV, 29.7\%) or severe COVID-19 (median ECV, 31.4\%) relative to healthy controls (median ECV 25\%, p <0.001) and that the prevalence of elevated ECV was $60 \%(24 / 60)$ in recovered COVID-19 patients [15]. Three studies on athlete participants reported a relatively lower prevalence of abnormal ECV (Rajpal et al.: 3.8\%, 1/26; Clark et al.: 4.5\%, 1/22; Malek et al.: 0\%, 0/26) than two studies on non-athletes (Eiros et al.: 37.4\%, Li et al.: 60\%) [16, 19, 37].

\section{Patterns of $L G E$, the involved segments of $L G E$, and $T 2$ abnormalities on CMR}

A non-ischemic LGE pattern was the most frequent pattern of myocardial LGE reported in 11 studies $(87.9 \%$, 123/140, Table 2) [12-14, 16, 18-20, 22, 28, 29, 37]. Specifically, subepicardial, epicardial, and mid-wall LGE were the patterns reported in these studies. Frequently reported myocardial LGE locations in eight studies included the mid and basal inferior, septal, and lateral segments [14-17, 19, 20, 29, 37].

Two studies reported eight locations of T2 abnormalities in six patients $[19,28]$. Similar to the LGE location, the mid-inferoseptum $(37.5 \%, 3 / 8)$ and midanteroseptum $(25 \%, 2 / 8)$ were the most common locations reported in the study by Rajpal et al. [19]. A study by Clark et al. on athletes reported that the $\mathrm{T} 2$ value was significantly higher in athletes who recovered from COVID-19 than healthy athlete controls $(p=0.02)$ [37].

\section{Ventricular systolic dysfunction on cine sequence}

Among the 16 included studies, six were excluded from the meta-analysis for ventricular dysfunction because the prevalence could not be extracted $[12,13,15,18,20,28]$.

\footnotetext{
(see figure on next page)

Fig. 2 Pooled prevalence of abnormal CMR findings in patients who recovered from COVID-19. a Pooled prevalence of total abnormal CMR findings. $\mathbf{b}$ Pooled prevalence of the diagnosis of myocarditis on CMR. c Pooled prevalence of pericardial late gadolinium enhancement (LGE). $\mathbf{d}$ Pooled prevalence of myocardial LGE. e Pooled prevalence of LGE (pericardial or myocardial). $\mathbf{f}$ Pooled prevalence of native T1 abnormality on the T1 map. g Pooled prevalence of T2 abnormality. $\mathbf{h}$ Pooled prevalence of LGE without T2 abnormality. i Pooled prevalence of T2 abnormality without LGE. j Pooled prevalence of pericardial effusion. $\mathbf{k}$ Pooled prevalence of ventricular systolic dysfunction. CMR, cardiovascular magnetic resonance; COVID-19, coronavirus disease 2019; LGE, late gadolinium enhancement
} 
a) Pooled prevalence of total abnormal CMR findings

$\begin{array}{lrr}\text { First author } & \text { Positive } & \text { Total } \\ & & \\ \text { Ng et al. } & 9 & 15 \\ \text { Huang et al. } & 15 & 26 \\ \text { Rajpal et al. } & 13 & 26 \\ \text { Clark et al. } & 4 & 59 \\ \text { Knight et al. } & 20 & 29 \\ \text { Puntmann et al. } & 78 & 100 \\ \text { Eiros et al. } & 104 & 139 \\ \text { Vago et al. } & 0 & 12 \\ \text { Brito et al. } & 26 & 48 \\ \text { Malek et al. } & 7 & 26 \\ \text { Li et al. } & 24 & 40 \\ \text { Starekova et al. } & 4 & 145 \\ \text { Wang et al. } & 13 & 44 \\ \text { Pan et al. } & 15 & 21 \\ \text { Zhou et al. } & 1 & 12 \\ \text { Kotecha et al. } & 80 & 148 \\ & & \\ \text { Fixed effect model } & & 890\end{array}$

Events per 100 observations

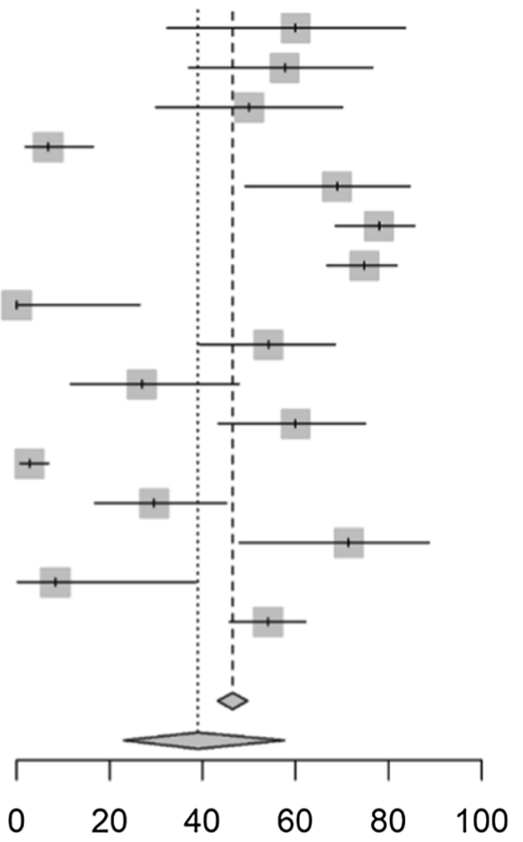

$\begin{array}{llllll}0 & 20 & 40 & 60 & 80 & 100\end{array}$

Prevalence

$95 \%-\mathrm{Cl}$

$60.00[32.29 ; 83.66]$

$57.69[36.92 ; 76.65]$

$50.00[29.93 ; 70.07]$

$6.78 \quad[1.88 ; 16.46]$

$68.97[49.17 ; 84.72]$

$78.00[68.61 ; 85.67]$

$74.82[66.76 ; 81.79]$

$0.00 \quad[0.00 ; 26.46]$

$54.17[39.17 ; 68.63]$

$26.92[11.57 ; 47.79]$

$60.00[43.33 ; 75.14]$

$2.76 \quad[0.76 ; 6.91]$

$29.55[16.76 ; 45.20]$

$71.43[47.82 ; 88.72]$

$8.33 \quad[0.21 ; 38.48]$

$54.05[45.68 ; 62.27]$

46.40 [43.15; 49.69]

38.90 [22.96; 57.63]

\section{Random effects model}

Heterogeneity: $I^{2}=95 \%, \chi_{15}^{2}=154.13(p<0.01)$

b) Pooled prevalence of the diagnosis of myocarditis on CMR

\section{First author}

$\mathrm{Ng}$ et al.

Huang et al.

Rajpal et al.

Clark et al.

Knight et al.

Eiros et al.

Vago et al.

Brito et al.

Malek et al.

Starekova et al.

Zhou et al.

Kotecha et al.

Fixed effect model

Random effects model

Heterogeneity: $I^{2}=93 \%, \chi_{11}^{2}=66.38(p<0.01)$

\section{Positive Total}

$\begin{array}{rr}4 & 15 \\ 8 & 26 \\ 4 & 26 \\ 2 & 59 \\ 13 & 29 \\ 51 & 139 \\ 0 & 12 \\ 0 & 48 \\ 0 & 26 \\ 2 & 145 \\ 0 & 12\end{array}$

12148

685
Events per 100 observations

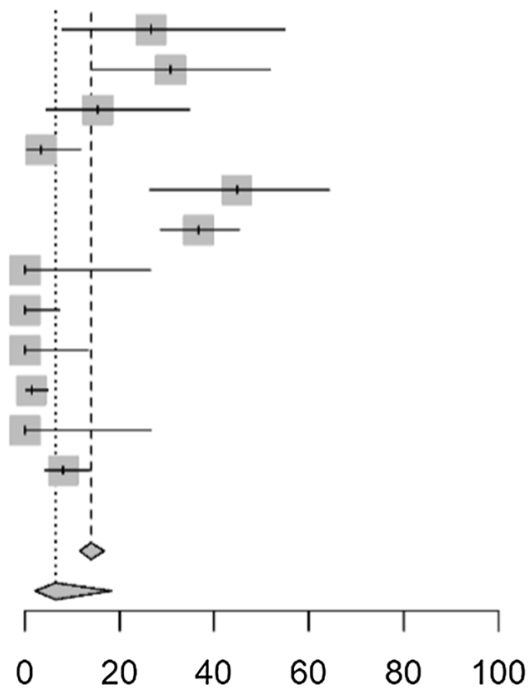

\section{Prevalence}

$26.67 \quad[7.79 ; 55.10]$

30.77 [14.33; 51.79]

$15.38 \quad[4.36 ; 34.87]$

$3.39 \quad[0.41 ; 11.71]$

$44.83[26.45 ; 64.31]$

$36.69[28.68 ; 45.28]$

$0.00 \quad[0.00 ; 26.46]$

$0.00 \quad[0.00 ; 7.40]$

$0.00 \quad[0.00 ; 13.23]$

$1.38 \quad[0.17 ; 4.89]$

$0.00 \quad[0.00 ; 26.46]$

8.11 [ 4.26; 13.73]

$14.01[11.61 ; 16.82]$

$6.46[2.07 ; 18.41]$ 
c) Pooled prevalence of pericardial late gadolinium enhancement (LGE)

First author

Huang et al.

Rajpal et al.

Clark et al.

Knight et al.

Puntmann et al.

Eiros et al.

Vago et al.

Brito et al.

Malek et al.

Li et al.

Starekova et al.

Wang et al.

Zhou et al.

Kotecha et al.

Fixed effect model

Random effects model

Heterogeneity: $I^{2}=94 \%, \chi_{13}^{2}=29.11(p<0.01)$

\section{Positive Total}

$\begin{array}{rr}0 & 26 \\ 0 & 26 \\ 1 & 59 \\ 0 & 29 \\ 22 & 100 \\ 0 & 139 \\ 0 & 12 \\ 19 & 48\end{array}$

$0 \quad 26$

040

1145

044

$\begin{array}{ll}0 & 12\end{array}$

$\begin{array}{ll}0 & 148\end{array}$

854
Events per 100

observations

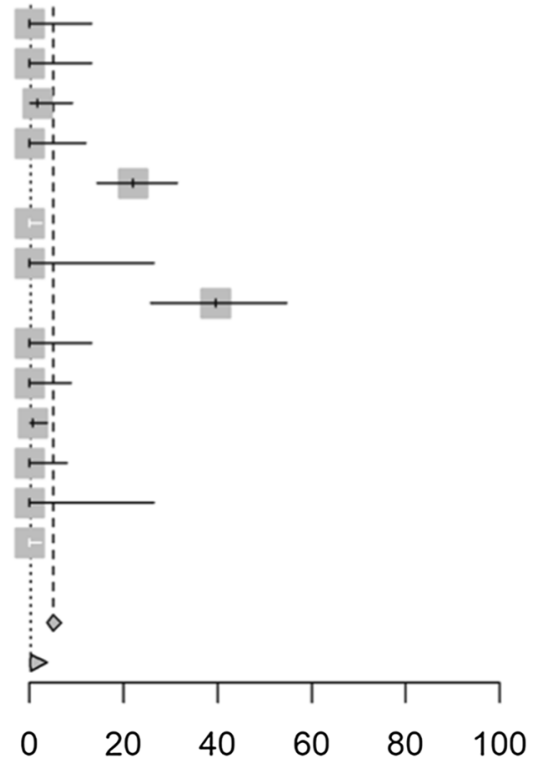

Prevalence $\quad 95 \%-\mathrm{Cl}$

$0.00 \quad[0.00 ; 13.23]$

$0.00 \quad[0.00 ; 13.23]$

$1.69 \quad[0.04 ; 9.09]$

$0.00 \quad[0.00 ; 11.94]$

$22.00[14.33 ; 31.39]$

$0.00 \quad[0.00 ; 2.62]$

$0.00[0.00 ; 26.46]$

$39.58[25.77 ; 54.73]$

$0.00[0.00 ; 13.23]$

$0.00 \quad[0.00 ; 8.81]$

$0.69 \quad[0.02 ; 3.78]$

$0.00 \quad[0.00 ; 8.04]$

$0.00 \quad[0.00 ; 26.46]$

$0.00 \quad[0.00 ; 2.46]$

$5.04 \quad[3.76 ; 6.72]$

$0.19[0.01 ; 3.74]$

d) Pooled prevalence of myocardial LGE

First author

$\mathrm{Ng}$ et al.

Huang et al.

Rajpal et al.

Clark et al.

Knight et al.

Puntmann et al.

Eiros et al.

Vago et al.

Brito et al.

Malek et al.

Li et al.

Starekova et al.

Wang et al.

Zhou et al.

Kotecha et al.

Fixed effect model

Random effects model

Positive Total

$\begin{array}{ll}3 & 15\end{array}$

826

$12 \quad 26$

359

$20 \quad 29$

$32 \quad 100$

$10 \quad 139$

$0 \quad 12$

148

126

140

$4 \quad 145$

$13 \quad 44$

$\begin{array}{ll}1 & 12\end{array}$

$\begin{array}{ll}70 & 144\end{array}$

865

Heterogeneity: $I^{2}=93 \%, \chi_{14}^{2}=135.62(p<0.01)$
Events per 100

observations
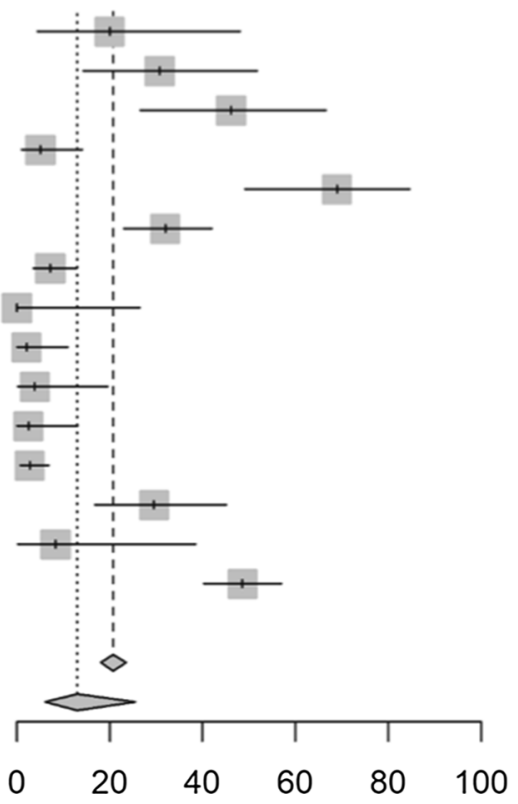

Prevalence $\quad 95 \%-\mathrm{Cl}$

20.00 [ 4.33; 48.09]

30.77 [14.33; 51.79]

$46.15[26.59 ; 66.63]$

$5.08 \quad[1.06 ; 14.15]$

$68.97[49.17 ; 84.72]$

$32.00[23.02 ; 42.08]$

$7.19 \quad[3.50 ; 12.83]$

$0.00 \quad[0.00 ; 26.46]$

$2.08 \quad[0.05 ; 11.07]$

$3.85[0.10 ; 19.64]$

$2.50 \quad[0.06 ; 13.16]$

$2.76 \quad[0.76 ; 6.91]$

$29.55[16.76 ; 45.20]$

8.33 [ $0.21 ; 38.48$ ]

$48.61[40.20 ; 57.08]$

20.69 [18.12; 23.52]

13.01 [ $6.13 ; 25.51]$

Fig. 2 continued 
e) Pooled prevalence of LGE (pericardial or myocardial)

\begin{tabular}{lrr} 
First author & Positive & Total \\
Ng et al. & 3 & 15 \\
Huang et al. & 8 & 26 \\
Rajpal et al. & 12 & 26 \\
Clark et al. & 4 & 59 \\
Knight et al. & 20 & 29 \\
Eiros et al. & 10 & 139 \\
Vago et al. & 0 & 12 \\
Malek et al. & 1 & 26 \\
Li et al. & 1 & 40 \\
Starekova et al. & 4 & 145 \\
Wang et al. & 13 & 44 \\
Zhou et al. & 1 & 12 \\
Kotecha et al. & 70 & 144 \\
& & \\
Fixed effect model & & 717 \\
Random effects model & \multicolumn{2}{l}{} \\
Heterogeneity: $I^{2}=92 \%, \chi_{12}^{2}=125.99$ & $(p<0.01)$
\end{tabular}

\section{Events per 100 \\ observations}

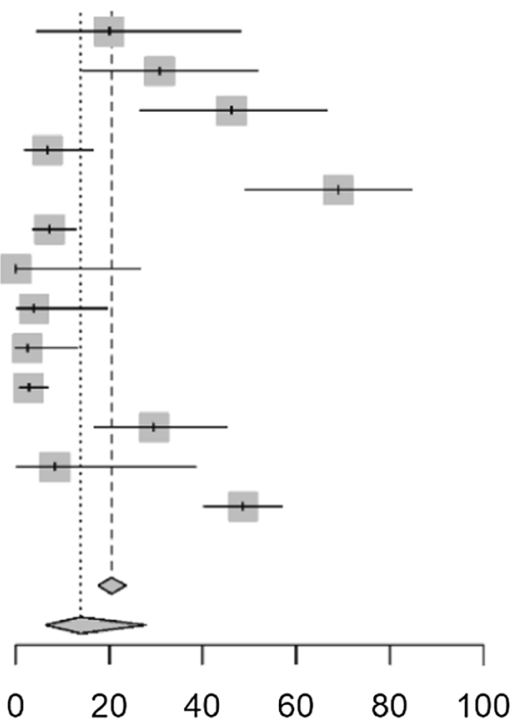

Prevalence

$95 \%-\mathrm{Cl}$

$20.00 \quad[4.33 ; 48.09]$

$30.77[14.33 ; 51.79]$

$46.15[26.59 ; 66.63]$

$6.78 \quad[1.88 ; 16.46]$

$68.97[49.17 ; 84.72]$

$7.19[3.50 ; 12.83]$

$0.00 \quad[0.00 ; 26.46]$

$3.85[0.10 ; 19.64]$

$2.50 \quad[0.06 ; 13.16]$

$2.76 \quad[0.76 ; 6.91]$

$29.55[16.76 ; 45.20]$

$8.33[0.21 ; 38.48]$

$48.61[40.20 ; 57.08]$

$20.50[17.70 ; 23.62]$

$13.89[6.32 ; 27.84]$

\section{f) Pooled prevalence of native T1 abnormality on the T1 map}

First author

$\mathrm{Ng}$ et al.

Rajpal et al.

Puntmann et al.

Eiros et al.

Vago et al.

Brito et al.

Malek et al.

Starekova et al.

Pan et al.

Kotecha et al.

Fixed effect model

Random effects model

Heterogeneity: $I^{2}=97 \%, \chi_{9}^{2}=104.23(p<0.01)$

\section{Positive Total}

$\begin{array}{rr}5 & 15 \\ 0 & 26 \\ 73 & 100\end{array}$

$\begin{array}{ll}58 & 139\end{array}$

$0 \quad 12$

948

$0 \quad 26$

2141

$\begin{array}{ll}5 & 21\end{array}$

$23 \quad 137$

665

Fig. 2 continued
Events per 100 observations

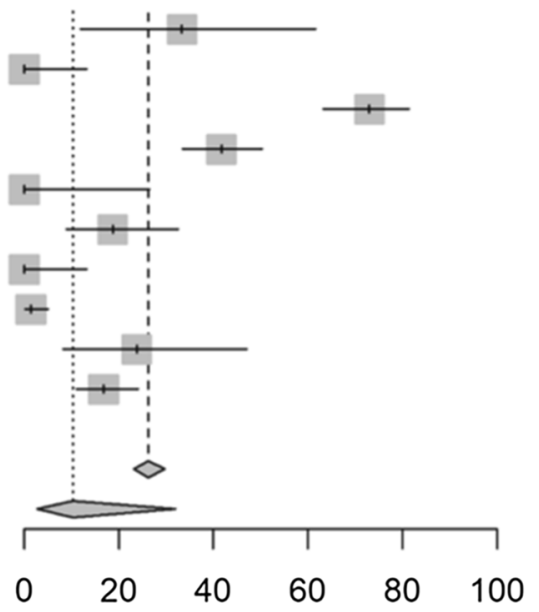

Prevalence

$95 \%-\mathrm{Cl}$

$33.33[11.82 ; 61.62]$

$0.00 \quad[0.00 ; 13.23]$

$73.00[63.20 ; 81.39]$

$41.73[33.43 ; 50.39]$

$0.00[0.00 ; 26.46]$

$18.75 \quad[8.95 ; 32.63]$

$0.00 \quad[0.00 ; 13.23]$

$1.42 \quad[0.17 ; 5.03]$

$23.81 \quad[8.22 ; 47.17]$

16.79 [10.95; 24.12]

26.32 [23.11; 29.80]

$10.36[2.75 ; 32.07]$
Four studies reported that significant RV dysfunction was observed in recovered COVID-19 patients [12, 17, 19, 37]. Huang et al. reported that the RV ejection fraction (RVEF) was significantly lower in recovered COVID-19 patients with abnormal CMR findings than in healthy controls (RVEF $36.5 \%$ vs. $46.1 \%, \mathrm{p}=0.01$ ). In contrast, the left ventricular (LV) ejection fraction (LVEF) was low in only one patient $(3.9 \%, 1 / 26)$ with abnormal CMR findings [12]. Pan et al. reported a decrease in RVEF in two patients (9.5\%), and the mean RVEF was significantly 


\section{g) Pooled prevalence of T2 abnormality}

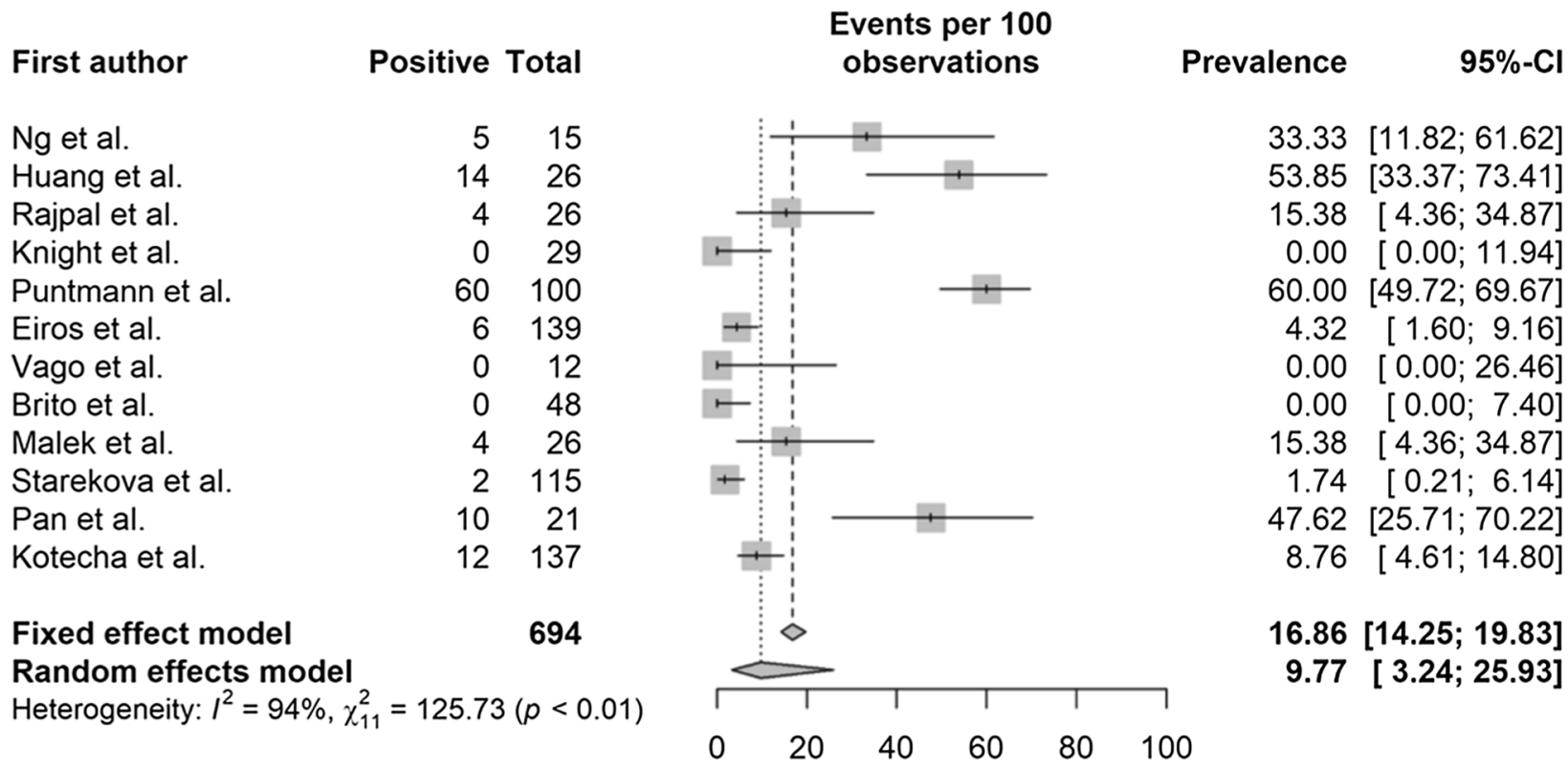

\section{h) Pooled prevalence of LGE without T2 abnormality}

\section{First author}

$\mathrm{Ng}$ et al.

Huang et al.

Rajpal et al.

Brito et al.

Malek et al.

Starekova et al.

Zhou et al.

Fixed effect model

Random effects model

Heterogeneity: $I^{2}=58 \%, \chi_{6}^{2}=16.72(p=0.01)$

\section{Positive Total}

$0 \quad 15$

126

$6 \quad 26$

148

126

2145

112

298

Fig. 2 continued
Events per 100 observations

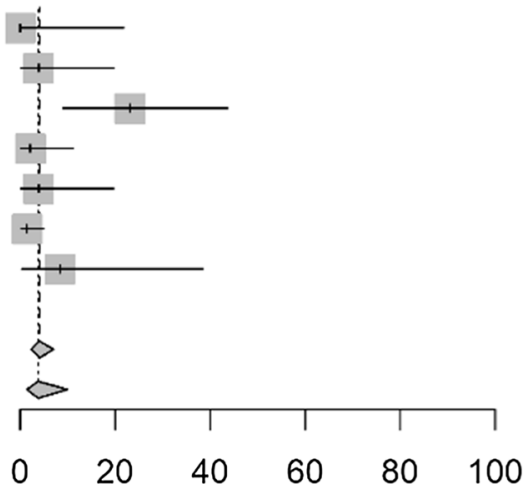

Prevalence $\quad 95 \%-\mathrm{Cl}$

$0.00[0.00 ; 21.80]$

$3.85[0.10 ; 19.64]$

$23.08[8.97 ; 43.65]$

$2.08[0.05 ; 11.07]$

$3.85[0.10 ; 19.64]$

$1.38[0.17 ; 4.89]$

$8.33[0.21 ; 38.48]$

$4.03[2.30 ; 6.96]$

$3.69[1.31 ; 9.97]$ lower in recovered COVID-19 patients than in controls $(\mathrm{p}<0.05)$. However, the mean LVEF was similar between recovered COVID-19 patients and controls [17].

LV or biventricular dysfunction in recovered COVID19 patients has been evaluated in previous studies [11, $13,18,21]$. Puntmann et al. measured and reported that the LVEF and RVEF were significantly lower in recovered COVID-19 patients than in matched controls (LVEF: 57\% vs. $62 \%$; RVEF: $54 \%$ vs. $59 \%$ ) (all, p<0.05) [18]. Malek et al. and Eiros et al. reported that the prevalence of LV systolic dysfunction in recovered COVID-19 patients was $8 \%$ and $5 \%$, respectively.

Malek et al. reported that two athletes (8\%) exhibited an enlarged LV with a slightly decreased LVEF, whereas RVEF was normal [16]. Although Eiros et al. reported LV wall motion abnormalities in seven patients $(5 \%$, 


\section{i) Pooled prevalence of $\mathrm{T} 2$ abnormality without LGE}

$\begin{array}{lrr}\text { First author } & \text { Positive } & \text { Total } \\ \text { Ng et al. } & 2 & 15 \\ \text { Huang et al. } & 7 & 26 \\ \text { Rajpal et al. } & 0 & 26 \\ \text { Knight et al. } & 0 & 29 \\ \text { Vago et al. } & 0 & 12 \\ \text { Brito et al. } & 0 & 48 \\ \text { Malek et al. } & 4 & 26 \\ \text { Starekova et al. } & 0 & 145 \\ & & \\ \text { Fixed effect model } & & 327 \\ \text { Random effects model } & \\ \text { Heterogeneity: } I^{2}=91 \%, \chi_{7}^{2}=1.52(p=0.98)\end{array}$

Events per 100 observations

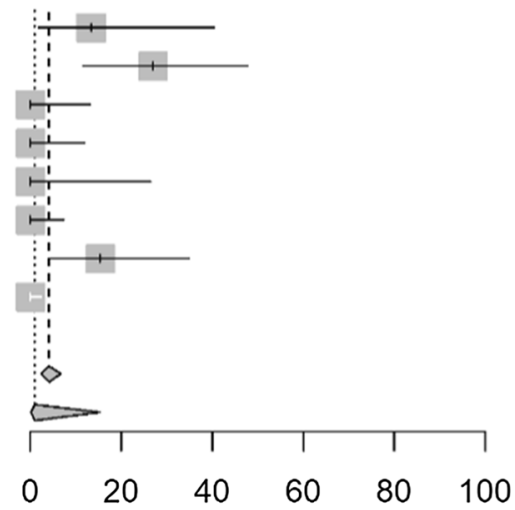

Prevalence $\quad 95 \%-\mathrm{Cl}$

$13.33[1.66 ; 40.46]$

$26.92[11.57 ; 47.79]$

$0.00[0.00 ; 13.23]$

$0.00[0.00 ; 11.94]$

$0.00[0.00 ; 26.46]$

$0.00 \quad[0.00 ; 7.40]$

$15.38 \quad[4.36 ; 34.87]$

$0.00 \quad[0.00 ; 2.51]$

$3.98 \quad[2.32 ; 6.73]$

$0.90[0.05 ; 15.32]$

\section{j) Pooled prevalence of pericardial effusion}

$\begin{array}{lrr}\text { First author } & \text { Positive } & \text { Total } \\ & 0 & 15 \\ \text { Ng et al. } & 7 & 26 \\ \text { Huang et al. } & 2 & 26 \\ \text { Rajpal et al. } & 2 & 29 \\ \text { Knight et al. } & 20 & 100 \\ \text { Puntmann et al. } & 42 & 139 \\ \text { Eiros et al. } & 0 & 12 \\ \text { Vago et al. } & 28 & 48 \\ \text { Brito et al. } & 2 & 26 \\ \text { Malek et al. } & 1 & 145 \\ \text { Starekova et al. } & 8 & 148 \\ \text { Kotecha et al. } & & \\ & & 714 \\ \text { Fixed effect model } & & \\ \text { Random effects model } \\ \text { Heterogeneity: } I^{2}=93 \%, \chi_{10}^{2}=76.83(p<0.01)\end{array}$

Events per 100 observations

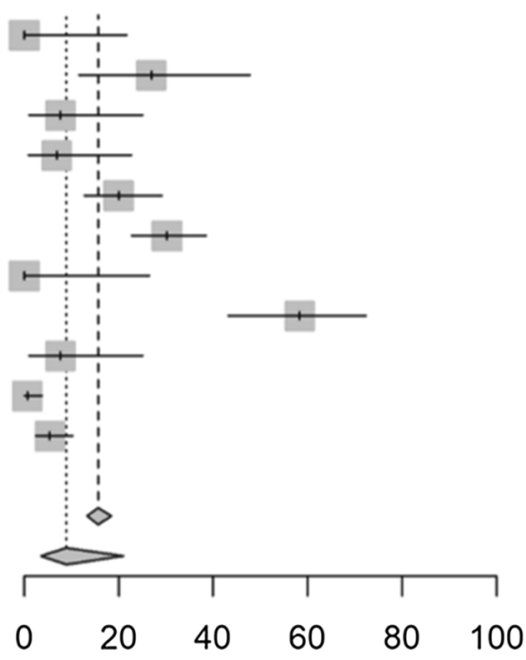

Prevalence

$95 \%-\mathrm{Cl}$

$0.00 \quad[0.00 ; 21.80]$

$26.92[11.57 ; 47.79]$

$7.69 \quad[0.95 ; 25.13]$

$6.90 \quad[0.85 ; 22.77]$

$20.00[12.67 ; 29.18]$

$30.22[22.72 ; 38.57]$

$0.00 \quad[0.00 ; 26.46]$

$58.33[43.21 ; 72.39]$

$7.69 \quad[0.95 ; 25.13]$

$0.69 \quad[0.02 ; 3.78]$

$5.41 \quad[2.36 ; 10.37]$

15.69 [13.20; 18.54]

$8.92[3.49 ; 20.97]$

Fig. 2 continued

7/139), data on RV function were not provided [11]. Although ventricular systolic function was normal, abnormal strain values were reported in two studies [15, 20]. Li et al. reported that global LV longitudinal strain was significantly lower in patients who recovered from moderate or severe COVID-19 than in healthy controls (moderate COVID-19 group:-12.5\%; severe COVID19 group: $-12.5 \%$; healthy controls: $-15.4 \% ; \mathrm{p}=0.002$ and $\mathrm{p}=0.001$, respectively) [15]. Wang et al. reported that recovered COVID-19 patients with LGE had significantly lower peak global circumferential strain values in the LV and RV and lower peak global longitudinal strain values in the RV than recovered COVID-19 patients with no LGE or healthy controls (both, $\mathrm{p}<0.05$ ) [20]. No cine abnormalities were reported in the populations studied by Vago et al., Ng et al. and Kotecha et al. [13, 14, 36]. 


\section{k) Pooled prevalence of ventricular systolic dysfunction.}

\section{First author}

Rajpal et al.

Clark et al.

Knight et al.

Eiros et al.

Vago et al.

Brito et al.

Malek et al.

Starekova et al.

Zhou et al.

Kotecha et al.

Fixed effect model

Random effects model

Heterogeneity: $I^{2}=62 \%, \chi_{9}^{2}=12.80(p=0.17)$

\section{Positive Total}

$\begin{array}{rr}1 & 26 \\ 0 & 59 \\ 2 & 29 \\ 7 & 139 \\ 0 & 12 \\ 1 & 48 \\ 0 & 26 \\ 2 & 145 \\ 0 & 12 \\ 17 & 148\end{array}$

Fig. 2 continued
Events per 100
observations

Prevalence $\quad 95 \%-\mathrm{Cl}$

$3.85[0.10 ; 19.64]$

$0.00[0.00 ; 6.06]$

$6.90[0.85 ; 22.77]$

$5.04[2.05 ; 10.10]$

$0.00[0.00 ; 26.46]$

$2.08[0.05 ; 11.07]$

$0.00[0.00 ; 13.23]$

$1.38[0.17 ; 4.89]$

$0.00[0.00 ; 26.46]$

$11.49[6.84 ; 17.75]$

$4.66[3.28 ; 6.58]$

$2.73[1.12 ; 6.47]$

Table 4 Meta-regression analysis for prevalence of each CMR finding

\begin{tabular}{|c|c|c|c|c|}
\hline \multirow[t]{2}{*}{ Parameter } & \multicolumn{2}{|c|}{$\begin{array}{l}\text { Univariable meta- } \\
\text { regression analysis }\end{array}$} & \multirow{2}{*}{$\begin{array}{l}\text { Multivariable meta- } \\
\text { regression analysis } \\
\text { p-value }\end{array}$} & \multirow{2}{*}{$\begin{array}{l}\text { Residual heterogeneity after } \\
\text { multivariable meta-regression } \\
\text { analysis } \\
\mathrm{I}^{2}\end{array}$} \\
\hline & p-value & $I^{2}$ & & \\
\hline Abnormal CMR findings & & & & $92.8 \%$ \\
\hline Athlete group & 0.002 & $93.4 \%$ & 0.018 & \\
\hline Undetermined cardiac enzyme level group & 0.061 & $94.6 \%$ & 0.173 & \\
\hline Diagnosis of myocarditis on CMR & & & & $\mathrm{N} / \mathrm{A}$ \\
\hline Athlete group & $<0.001$ & $90.6 \%$ & N/A & \\
\hline Undetermined cardiac enzyme level group & 0.405 & $93.7 \%$ & N/A & \\
\hline Presence of myocardial LGE & & & & $93.9 \%$ \\
\hline Athlete group & 0.050 & $95.4 \%$ & 0.206 & \\
\hline Undetermined cardiac enzyme level group & 0.033 & $94.0 \%$ & 0.138 & \\
\hline T2 abnormality & & & & N/A \\
\hline Athlete group & 0.035 & $97.2 \%$ & N/A & \\
\hline Undetermined cardiac enzyme level group & 0.629 & $96.9 \%$ & N/A & \\
\hline Pericardial effusion & & & & N/A \\
\hline Athlete group & 0.753 & $93.8 \%$ & N/A & \\
\hline Undetermined cardiac enzyme level group & 0.353 & $92.1 \%$ & N/A & \\
\hline
\end{tabular}

CMR cardiovascular magnetic resonance, $N A$ not available

\section{Publication bias}

Funnel plots of the prevalence values of abnormal CMR findings, a diagnosis of myocarditis on CMR, myocardial LGE, a T2 abnormality, and pericardial effusion are presented in Additional file 1: Fig. S1. All parameters had symmetric funnel plots without significant publication bias ( $>>0.05)$, except for T2 abnormality without LGE $(\mathrm{p}=0.04)$. 


\section{Discussion}

This meta-analysis revealed that nearly half of the patients exhibited one or more abnormal CMR findings after recovery from COVID-19. Athletes and patients in the normal cardiac enzyme level subgroups showed a lower prevalence of abnormal CMR findings than non-athletes and patients in the undetermined cardiac enzyme level subgroups. The most frequent abnormal CMR finding was the presence of an elevated native T1 value on the T1 map (26.3\%), followed by a presence of myocardial LGE (20.7\%).

Non-invasive CMR is a valuable diagnostic tool to evaluate the presence and extent of myocardial injury in COVID-19 patients [9]. A previously published systematic review reported CMR findings for 199 COVID-19 patients, including patients with myocarditis (40.2\%), myopericarditis, stress-induced cardiomyopathy, and ischemia [10]. However, the studies included in this systematic review primarily conducted CMR during the active phase of COVID-19 [10]. Therefore, the data did not contribute to our understanding of whether myocardial inflammation or scarring would be observed on CMR in recovered COVID-19 patients.

Patients with myocarditis may develop arrhythmia or heart failure after recovery due to residual myocardial fibrosis or scarring [7]. LGE with T2 abnormality on CMR suggests that myocardial edema is present and the myocarditis is in the acute inflammatory phase. Consequently, the extent of LGE can diminish after recovery [38]. In contrast, LGE without a T2 abnormality after recovery from myocarditis indicates myocardial scarring or fibrosis and is associated with a poorer prognosis [9, 39]. The prevalence of LGE in myocarditis patients other than COVID-19 dropped from 72 to $48 \%$ and that of a T2 abnormality decreased from 57 to $7 \%$ at 12 months follow-up in a previous study [38].

The time interval between a diagnosis of COVID19 and CMR varied among the studies included in this meta-analysis. Nevertheless, CMR was performed within 22 weeks of COVID-19 diagnosis, a shorter interval than that reported in previous studies on non-COVID-19 myocarditis [38]. The pooled prevalence of CMR findings of acute myocarditis in recovered COVID-19 patients diagnosed with myocarditis (14.0\%), elevated native $\mathrm{T} 1$ (26.3\%), myocardial LGE (20.7\%) and T2 abnormality (16.9\%) was higher than that of myocardial LGE without T2 abnormality (4.0\%), which indicates permanent myocardial scarring and is associated with a poor prognosis. A mid-wall septal pattern of LGE, a poor prognostic factor in non-COVID-19 myocarditis, has been reported in several studies $[14,16,20,28]$. These results suggest that active myocardial inflammation persists in the early phase of recovery from COVID-19. Therefore, the results of large-scale, ongoing studies (C-MORE, CISCO-19 and COVID-HEART) with long-term follow-up may address whether these findings will disappear or remain as permanent myocardial fibrosis [40-42].

Myocarditis in athletes can be critical because athletes place themselves at a higher risk of sudden cardiac death or adverse cardiac events during strenuous exercise [25]. Currently, the consensus among experts does not recommend routine CMR for evaluating whether to allow athletes who recovered from COVID-19 to return to play [43-46]. Typically, CMR is not a firstline modality for evaluating patients with suspected myocardial injuries. Instead, CMR is performed after electrocardiography, cardiac biomarker analysis, or transthoracic echocardiography to provide a more advanced and comprehensive evaluation in patients with ongoing clinical concerns [43-46]. Although the prevalence of abnormal CMR findings was lower in athletes than in non-athletes in this meta-analysis, the prevalence of LGE without T2 abnormality was similar between the two groups. Moreover, the prevalence of pericardial LGE was higher in athletes than in nonathletes. Therefore, long-term follow-up studies with larger numbers of participants (athletes) who recovered from COVID-19 are necessary to determine the significance of LGE observed on CMR.

In this meta-analysis, we observed that patients with normal cardiac enzyme levels had less frequent CMR abnormalities than patients with unknown cardiac enzyme levels (59.4\% vs. 35.9\%). Although our meta-analysis could not include a subgroup analysis for patients with elevated cardiac enzymes, elevated troponin levels are well-established markers of myocardial injury. High troponin levels are associated with severe disease and a poor prognosis in COVID-19 patients [47, 48]. Elevated troponin levels in recovered COVID-19 patients suggests ongoing subclinical inflammation; however, it is uncertain whether normal cardiac enzyme levels indicate an absence of myocardial scars. CMR may provide risk stratification for patients who recovered from COVID-19.

Besides myocardial abnormality, ventricular systolic dysfunction and pericardial abnormalities have also been reported in recovered COVID-19 patients. RV systolic dysfunction is the most common cine abnormality in recovered COVID-19 patients and is associated with increased pulmonary vascular resistance [49], acute respiratory distress syndrome, and poor outcomes in patients with COVID-19 [50]. Although the prevalence of functional abnormalities is low relative to those observed for other CMR parameters, studies clarifying the mechanism underlying the restoration of cardiac function in these patients are needed. This meta-analysis revealed that pericardial effusion was frequently 
observed in recovered COVID-19 patients, whereas pericardial LGE was relatively rare. Pericarditis, pericardial effusion, and cardiac tamponade have occasionally been reported during the active phase of COVID-19 [51, 52]; however, the underlying mechanisms remain unclear. Inadequate immune response to COVID-19 may lead to slower clearance of the virus from the peri-myocardium, development of pericarditis secondary to myocardial inflammation, or pericardial effusion caused by generalized COVID-19-related multi-systemic inflammatory syndrome [13, 18, 21]. The outcome of this evidence is unknown; however, our study findings would support further study.

Comprehensive and definitive cardiac imaging guidelines for recovered COVID-19 patients, especially the non-athlete population, are lacking. Future large-scale, long-term studies may reveal the clinical significance of abnormal CMR findings. Based on our study and future studies, appropriate surveillance guidelines for using CMR and other cardiac imaging modalities in recovered COVID-19 patients should be established.

\section{Limitations}

Our study has several limitations. First, the subgroup of patients with elevated cardiac enzyme levels could not be analyzed due to the small number of studies and patients. Second, an analysis of ventricular systolic dysfunction in the subgroup of patients with normal cardiac enzyme levels was not conducted due to the small number of patients with ventricular systolic dysfunction. Third, certain data necessary for subgroup analysis, such as the presence of cardiac symptoms or underlying cardiac disease, or abnormalities revealed on electrocardiography or echocardiography, could not be extracted. Lastly, CMR scans were performed within 22 weeks of COVID-19 recovery, and longer-term studies are needed to determine the clinical significance of these findings.

\section{Conclusions}

Nearly half of those recovering from COVID-19 exhibit one or more abnormal CMR findings. The prevalence of abnormal CMR findings was lower in athletes and patients with normal cardiac enzyme levels than in nonathletes and patients with undetermined cardiac enzyme levels. We propose that comprehensive surveillance with CMR could help stratify the risks of cardiovascular complications in recovered COVID-19 patients.

\footnotetext{
Abbreviations

Cl: Confidence interval; CMR: Cardiovascular magnetic resonance; COVID-19: Coronavirus disease 2019; CRP: C reactive protein; ECG: Electrocardiogram; ECV: Extracellular volume; LGE: Late gadolinium enhancement; LV: Left
}

ventricle/left ventricular; LVEF: Left ventricular ejection fraction; RV: Right ventricle/right ventricular; RVEF: Right ventricular ejection fraction; T2W: T2-weighted.

\section{Supplementary Information}

The online version contains supplementary material available at https://doi. org/10.1186/s12968-021-00792-7.

Additional file 1. Supplementary Table 1. Quality assessment of the included studies. Supplemental Appendix 1. Search terms used in PubMed, the Cochrane library and EMBASE. Supplementary Figure 1. Funnel plots to detect publication bias.

\section{Acknowledgements}

Not applicable.

\section{Authors' contributions}

JYK analyzed and interpretated data, drafted the manuscript, and approved the final manuscript submitted. $\mathrm{HH}$ analyzed and interpretated data, drafted and revised the manuscript, and approved the final manuscript submitted. YJS concepted and designed or analyzed and interpretated data, drafted and revised the manuscript, and approved the final manuscript submitted. All authors read and approved the final manuscript.

Funding

This research was supported by the Bisa Research Grant of Keimyung University in 2020.

Availability of data and materials

The dataset analyzed during the current study are available from the corresponding author on reasonable request.

\section{Declarations}

Ethics approval and consent to participate

Not applicable.

Consent for publication

Not applicable.

\section{Competing interests}

The authors declare that they have no competing interests.

\section{Author details}

${ }^{1}$ Department of Radiology, Dongsan Hospital, Keimyung University College of Medicine, Daegu, Korea. ${ }^{2}$ Department of Radiology, Research Institute of Radiological Science, Severance Hospital, Yonsei University College of Medicine, 50-1 Yonsei-ro, Seodaemun-gu, Seoul 03722, Korea.

Received: 29 March 2021 Accepted: 8 July 2021

Published online: 03 September 2021

\section{References}

1. Organization WH. WHO Director-General's opening remarks at the media briefing on COVID-19 - 11 March 2020. 2020.

2. World Health Organization. WHO Coronavirus Disease (COVID-19) Dashboard. Accessed 7 Jul 2021.

3. Bansal M. Cardiovascular disease and COVID-19. Diabetes Metab Syndr. 2020;14(3):247-50. https://doi.org/10.1016/j.dsx.2020.03.013.

4. Driggin E, Madhavan MV, Bikdeli B, Chuich T, Laracy J, Biondi-Zoccai G, et al. Cardiovascular considerations for patients, health care workers, and health systems during the COVID-19 pandemic. J Am Coll Cardiol. 2020;75(18):2352-71. https://doi.org/10.1016/j.jacc.2020.03.031. 
5. Shi S, Qin M, Shen B, Cai Y, Liu T, Yang F, et al. Association of cardiac injury with mortality in hospitalized patients with COVID-19 in Wuhan, China. JAMA Cardiol. 2020. https://doi.org/10.1001/jamacardio.2020.0950.

6. Santoso A, Pranata R, Wibowo A, Al-Farabi MJ, Huang I, Antariksa B. Cardiac injury is associated with mortality and critically ill pneumonia in COVID-19: a meta-analysis. Am J Emerg Med. 2020. https://doi.org/10. 1016/j.ajem.2020.04.052.

7. Peretto G, Sala S, Rizzo S, De Luca G, Campochiaro C, Sartorelli S, et al. Arrhythmias in myocarditis: state of the art. Heart Rhythm. 2019;16(5):793-801. https://doi.org/10.1016/j.hrthm.2018.11.024.

8. Kearney MT, Cotton JM, Richardson PJ, Shah AM. Viral myocarditis and dilated cardiomyopathy: mechanisms, manifestations, and management. Postgrad Med J. 2001;77(903):4-10. https://doi.org/10.1136/pmj.77.903.4.

9. Ferreira VM, Schulz-Menger J, Holmvang G, Kramer CM, Carbone I, Sechtem $U$, et al. Cardiovascular magnetic resonance in nonischemic myocardial inflammation. Exp Recommend. 2018;72(24):3158-76. https:// doi.org/10.1016/j.jacc.2018.09.072\%JJournaloftheAmericanCollegeof Cardiology.

10. Ojha V, Verma M, Pandey NN, Mani A, Malhi AS, Kumar S, et al. Cardiac magnetic resonance imaging in coronavirus disease 2019 (COVID-19): a systematic review of cardiac magnetic resonance imaging findings in 199 patients. J Thorac Imaging. 2021;36(2):73-83. https://doi.org/10.1097/rti. 0000000000000574.

11. Eiros R, Barreiro-Perez M, Martin-Garcia A, Almeida J, Villacorta E, PerezPons A, et al. Pericarditis and myocarditis long after SARS-CoV-2 infection: a cross-sectional descriptive study in health-care workers. medRxiv. 2020:2020.07.12.20151316. doi: https://doi.org/10.1101/2020.07.12.20151 316.

12. Huang L, Zhao P, Tang D, Zhu T, Han R, Zhan C, et al. Cardiac involvement in patients recovered from COVID-2019 identified using magnetic resonance imaging. JACC Cardiovasc Imag. 2020;13(11):2330-9. https:// doi.org/10.1016/j.jcmg.2020.05.004.

13. Knight DS, Kotecha T, Razvi Y, Chacko L, Brown JT, Jeetley PS, et al. COVID-19: myocardial injury in survivors. Circulation. 2020;142(11):1120-2. https://doi.org/10.1161/circulationaha.120. 049252.

14. Kotecha T, Knight DS, Razvi Y, Kumar K, Vimalesvaran K, Thornton G, et al. Patterns of myocardial injury in recovered troponin-positive COVID-19 patients assessed by cardiovascular magnetic resonance. Eur Heart J. 2021. https://doi.org/10.1093/eurheartj/ehab075.

15. Li X, Wang H, Zhao R, Wang T, Zhu Y, Qian Y, et al. Elevated extracellular volume fraction and reduced global longitudinal strains in patients recovered from COVID-19 without clinical cardiac findings. Radiology. 2021;2021:203998. https://doi.org/10.1148/radiol.2021203998.

16. Małek ŁA, Marczak M, Miłosz-Wieczorek B, Konopka M, Braksator W, Drygas $W$, et al. Cardiac involvement in consecutive elite athletes recovered from Covid-19: a magnetic resonance study. J Magn Reson Imaging. 2021. https://doi.org/10.1002/jmri.27513.

17. Pan C, Zhang Z, Luo L, Wu W, Jia T, Lu L, et al. Cardiac T1 and T2 mapping showed myocardial involvement in recovered covid-19 patients initially considered devoid of cardiac damage. J Magn Reson Imaging 2021. https://doi.org/10.1002/jmri.27534.

18. Puntmann VO, Carerj ML, Wieters I, Fahim M, Arendt C, Hoffmann J, et al. Outcomes of cardiovascular magnetic resonance imaging in patients recently recovered from coronavirus disease 2019 (COVID-19). JAMA Cardiol. 2020;5(11):1265-73. https://doi.org/10.1001/jamacardio. 2020.3557.

19. Rajpal S, Tong MS, Borchers J, Zareba KM, Obarski TP, Simonetti OP, et al. Cardiovascular magnetic resonance findings in competitive athletes recovering from COVID-19 infection. JAMA Cardiol. 2021;6(1):116-8. https://doi.org/10.1001/jamacardio.2020.4916.

20. Wang H, Li R, Zhou Z, Jiang H, Yan Z, Tao X, et al. Cardiac involvement in COVID-19 patients: mid-term follow up by cardiovascular magnetic resonance. J Cardiovasc Magn Reson. 2021;23(1):14. https://doi.org/10. 1186/s12968-021-00710-X.

21. Brito D, Meester S, Yanamala N, Patel HB, Balcik BJ, Casaclang-Verzosa $G$, et al. high prevalence of pericardial involvement in college student athletes recovering from COVID-19. JACC Cardiovasc Imaging. 2021;14(3):541-55. https://doi.org/10.1016/j.jcmg.2020.10.023.

22. Ng MY, Ferreira VM, Leung ST, Yin Lee JC, Ho-Tung Fong A, To Liu RW, et al. Patients recovered from COVID-19 show ongoing subclinical myocarditis as revealed by cardiac magnetic resonance imaging. JACC Cardiovasc Imaging. 2020;13(11):2476-8. https://doi.org/10.1016/j. jcmg.2020.08.012.

23. Babu-Narayan SV, McCarthy KP, Ho SY, Magee AG, Kilner PJ, Sheppard MN. Myocarditis and sudden cardiac death in the young. Circulation. 2007;116(6):e122-5. https://doi.org/10.1161/CIRCULATIONAHA.107. 693085.

24. di Gioia CR, Giordano C, Cerbelli B, Pisano A, Perli E, De Dominicis E, et al. Nonischemic left ventricular scar and cardiac sudden death in the young. Hum Pathol. 2016;58:78-89. https://doi.org/10.1016/j.humpath. 2016.08.004.

25. Eichhorn C, Bière L, Schnell F, Schmied C, Wilhelm M, Kwong RY, et al. Myocarditis in athletes is a challenge: diagnosis, risk stratification, and uncertainties. JACC Cardiovasc Imaging. 2020;13(2 Pt 1):494-507. https://doi.org/10.1016/j.jcmg.2019.01.039.

26. McInnes MDF, Moher D, Thombs BD, McGrath TA, Bossuyt PM, Clifford T, et al. preferred reporting items for a systematic review and meta-analysis of diagnostic test accuracy studies: the PRISMA-DTA statement. JAMA. 2018;319(4):388-96. https://doi.org/10.1001/jama.2017.19163.

27. van de Schoor FR, Aengevaeren VL, Hopman MTE, Oxborough DL, George KP, Thompson PD, et al. Myocardial fibrosis in athletes. Mayo Clin Proc. 2016;91(11):1617-31. https://doi.org/10.1016/j.mayocp.2016. 07.012

28. Starekova J, Bluemke DA, Bradham WS, Eckhardt LL, Grist TM, Kusmirek $\mathrm{JE}$, et al. Evaluation for myocarditis in competitive student athletes recovering from coronavirus disease with cardiac magnetic resonance imaging. JAMA Cardiol. 2019. https://doi.org/10.1001/jamacardio.2020. 7444 .

29. Zhou M, Wong CK, Un KC, Lau YM, Lee JC, Tam FC, et al. Cardiovascular sequalae in uncomplicated COVID-19 survivors. PLOS ONE. 2021;16(2): e0246732. https://doi.org/10.1371/journal.pone.0246732.

30. Wells BS, O'Connell D, Peterson J, Welch V, Losos M, Tugwell P. The Newcastle-Ottawa Scale (NOS) for assessing the quality of nonrandomised studies in meta-analyses. Berlin: Springer; 2017.

31. Higgins JP, Thompson SG. Quantifying heterogeneity in a meta-analysis. Stat Med. 2002;21(11):1539-58. https://doi.org/10.1002/sim.1186.

32. DerSimonian R, Laird N. Meta-analysis in clinical trials. Control Clin Trials. 1986;7(3):177-88. https://doi.org/10.1016/0197-2456(86)90046-2.

33. Egger M, Davey Smith G, Schneider M, Minder C. Bias in meta-analysis detected by a simple, graphical test. BMJ. 1997;315(7109):629-34. https://doi.org/10.1136/bmj.315.7109.629.

34. Viechtbauer W. Conducting meta-analyses in R with the metafor package. PLoS ONE. 2010;36:88.

35. Balduzzi S, Rücker G, Schwarzer G. How to perform a meta-analysis with R: a practical tutorial. Evid Based Ment Health. 2019;22(4):153-60. https://doi.org/10.1136/ebmental-2019-300117.

36. Vago H, Szabo L, Dohy Z, Merkely B. Cardiac magnetic resonance findings in patients recovered from COVID-19: Initial experiences in elite athletes. JACC Cardiovasc Imaging. 2020. https://doi.org/10.1016/j. jcmg.2020.11.014.

37. Clark DE, Parikh A, Dendy JM, Diamond AB, George-Durrett K, Fish FA et al. COVID-19 myocardial pathology evaluation in athletes with cardiac magnetic resonance (COMPETE CMR). Circulation. 2020. https:// doi.org/10.1161/circulationaha.120.052573.

38. White JA, Hansen R, Abdelhaleem A, Mikami Y, Peng M, Rivest S, et al. Natural history of myocardial injury and chamber remodeling in acute myocarditis. Circulation. 2019;12(7):e008614. https://doi.org/10.1161/ CIRCIMAGING.118.008614.

39. Aquaro GD, Ghebru Habtemicael Y, Camastra G, Monti L, Dellegrottaglie S, Moro C, et al. Prognostic value of repeating cardiac magnetic resonance in patients with acute myocarditis. J Am Coll Cardiol. 2019;74(20):2439-48. https://doi.org/10.1016/j.jacc.2019.08.1061.

40. ClinicalTrials.gov. U.S. National Library of Medicine. Capturing MultiORgan Effects of COVID-19 (C-MORE) https://clinicaltrials.gov/ct2/show/ NCT04510025. Accessed 04 June 2021.

41. U.S. National Library of Medicine. Cardiac Imaging in SARS-CoV-2 (COVID-19) (CISCO-19). https://clinicaltrials.gov/ct2/show/NCT04 403607. Accessed 04 June 2021.

42. Gorecka M, McCann GP, Berry C, Ferreira VM, Moon JC, Miller CA, et al. Demographic, multi-morbidity and genetic impact on myocardial involvement and its recovery from COVID-19: protocol 
design of COVID-HEART—a UK, multicentre, observational study. J Cardiovasc Magn Reson. 2021;23(1):77. https://doi.org/10.1186/ s12968-021-00752-1.

43. Phelan D, Kim JH, Elliott MD, Wasfy MM, Cremer P, Johri AM, et al. Screening of potential cardiac involvement in competitive athletes recovering from COVID-19: an expert consensus statement. JACC Cardiovasc Imaging. 2020;13(12):2635-52. https://doi.org/10.1016/j.jcmg. 2020.10.005.

44. Dores H, Cardim N. Return to play after COVID-19: a sport cardiologist's view. Br J Sports Med. 2020;54(19):1132-3. https://doi.org/10.1136/ bjsports-2020-102482.

45. McKinney J, Connelly KA, Dorian P, Fournier A, Goodman JM, Grubic N, et al. COVID-19-Myocarditis and return-to-play: reflections and recommendations from a canadian working group. Can J Cardiol. 2020. https://doi.org/10.1016/j.cjca.2020.11.007.

46. Kim JH, Levine BD, Phelan D, Emery MS, Martinez MW, Chung EH, et al. Coronavirus disease and the athletic heart: emerging perspectives on pathology, risks, and return to play. JAMA Cardiol. 2019. https://doi.org/ 10.1001/jamacardio.2020.5890.

47. Sandoval Y, Januzzi JL Jr, Jaffe AS. Cardiac troponin for assessment of myocardial injury in COVID-19: JACC review topic of the week. J Am Coll Cardiol. 2020;76(10):1244-58. https://doi.org/10.1016/j.jacc.2020. 06.068
48. Lombardi CM, Carubelli V, Iorio A, Inciardi RM, Bellasi A, Canale C, et al. Association of troponin levels with mortality in italian patients hospitalized with coronavirus disease 2019: results of a multicenter study. JAMA Cardiol. 2020;5(11):1274-80. https://doi.org/10.1001/jamacardio. 2020.3538.

49. Park S, Lee SM, Lee KH, Jung KH, Bae W, Choe J, et al. Deep learningbased detection system for multiclass lesions on chest radiographs: comparison with observer readings. Eur Radiol. 2020;30(3):1359-68. https://doi.org/10.1007/s00330-019-06532-x.

50. Bleakley C, Singh S, Garfield B, Morosin M, Surkova E, Mandalia MS, et al. Right ventricular dysfunction in critically ill COVID-19 ARDS. Int J Cardiol. 2020. https://doi.org/10.1016/j.ijcard.2020.11.043.

51. Hua A, O'Gallagher K, Sado D, Byrne J. Life-threatening cardiac tamponade complicating myo-pericarditis in COVID-19. Eur Heart J. 2020;41(22):2130. https://doi.org/10.1093/eurheartj/ehaa253.

52. Dabbagh MF, Aurora L, D'Souza P, Weinmann AJ, Bhargava P, Basir MB. Cardiac tamponade secondary to COVID-19. JACC Case Rep. 2020;2(9):1326-30. https://doi.org/10.1016/j.jaccas.2020.04.009.

\section{Publisher's Note}

Springer Nature remains neutral with regard to jurisdictional claims in published maps and institutional affiliations.
Ready to submit your research? Choose BMC and benefit from:

- fast, convenient online submission

- thorough peer review by experienced researchers in your field

- rapid publication on acceptance

- support for research data, including large and complex data types

- gold Open Access which fosters wider collaboration and increased citations

- maximum visibility for your research: over $100 \mathrm{M}$ website views per year

At $\mathrm{BMC}$, research is always in progress.

Learn more biomedcentral.com/submissions 